OPEN ACCESS

Edited by:

Vicente Herranz-Pérez,

University of Valencia, Spain

Reviewed by:

Goichi Miyoshi,

Tokyo Women's Medical University,

Japan

Vivian Capilla-González,

Andalusian Center of Molecular Biology and Regenerative Medicine

(CABIMER), Spain

*Correspondence:

Shigeyuki Esum

esumi@kumamoto-u.ac.jp

Specialty section:

This article was submitted to

Neurodegeneration,

a section of the journal

Frontiers in Neuroscience

Received: 18 September 2020

Accepted: 10 June 2021

Published: 08 July 2021

Citation:

Esumi S, Nasu M, Kawauchi T, Miike K, Morooka K, Yanagawa Y, Seki T, Sakimura K, Fukuda T and Tamamaki N (2021) Characterization

and Stage-Dependent Lineage Analysis of Intermediate Progenitors of Cortical GABAergic Interneurons.

Front. Neurosci. 15:607908.

doi: 10.3389/fnins.2021.607908

\section{Characterization and Stage-Dependent Lineage Analysis of Intermediate Progenitors of Cortical GABAergic Interneurons}

\author{
Shigeyuki Esumi 1*, Makoto Nasu², Takeshi Kawauchi ${ }^{3}$, Koichiro Miike ${ }^{4}$, Kento Morooka ${ }^{5}$, \\ Yuchio Yanagawa ${ }^{6}$, Tatsunori Seki ${ }^{7}$, Kenji Sakimura ${ }^{8}$, Takaichi Fukuda ${ }^{1}$ and \\ Nobuaki Tamamaki ${ }^{9}$
}

1 Department of Anatomy and Neurobiology, Graduate School of Medical Sciences, Kumamoto University, Kumamoto, Japan, ${ }^{2}$ Department of Health Sciences, Faculty of Life Sciences, Kumamoto University, Kumamoto, Japan, ${ }^{3}$ Laboratory of Molecular Life Science, Institute of Biomedical Research and Innovation, Foundation for Biomedical Research and Innovation at Kobe (FBRI), Kobe, Japan, ${ }^{4}$ Institute of Molecular Embryology and Genetics, Kumamoto University, Kumamoto, Japan, ${ }^{5}$ Kumamoto University Hospital, Kumamoto, Japan, ${ }^{6}$ Department of Genetic and Behavioral Neuroscience, Gunma University Graduate School of Medicine, Maebashi, Japan, ${ }^{7}$ Department of Histology and Neuroanatomy, Tokyo Medical University, Tokyo, Japan, ${ }^{8}$ Department of Cellular Neurobiology, Brain Research Institute, Niigata University, Niigata, Japan, ${ }^{9}$ Department of Morphological Neural Science, Graduate School of Life Sciences, Kumamoto University, Kumamoto, Japan

Intermediate progenitors of both excitatory and inhibitory neurons, which can replenish neurons in the adult brain, were recently identified. However, the generation of intermediate progenitors of GABAergic inhibitory neurons (IPGNs) has not been studied in detail. Here, we characterized the spatiotemporal distribution of IPGNs in mouse cerebral cortex. IPGNs generated neurons during both embryonic and postnatal stages, but the embryonic IPGNs were more proliferative. Our lineage tracing analyses showed that the embryonically proliferating IPGNs tended to localize to the superficial layers rather than the deep cortical layers at 3 weeks after birth. We also found that embryonic IPGNs derived from the medial and caudal ganglionic eminence (CGE) but more than half of the embryonic IPGNs were derived from the CGE and broadly distributed in the cerebral cortex. Taken together, our data indicate that the broadly located IPGNs during embryonic and postnatal stages exhibit a different proliferative property and layer distribution.

Keywords: GABAergic neuron progenitors, lineage, cortical development, fate analysis, laminar distribution

\section{INTRODUCTION}

Brain function relies on the concordance between excitatory and inhibitory neuron activities, and excitatory-inhibitory imbalances are associated with many psychiatric disorders (Bozzi et al., 2012; Marín, 2012; Lim et al., 2018; Sohal and Rubenstein, 2019). Excitatory and inhibitory neurons are generated in different brain regions. In the developing cerebral cortex, glutamatergic excitatory neurons originate from the dorsal ventricular zone (VZ) of the cerebral cortex, whereas GABAergic inhibitory neurons (IPGNs) arise from the medial ganglionic eminence (MGE), caudal ganglionic eminence (CGE), or preoptic area (POA) and migrate tangentially to the cerebral cortex 
(Anderson et al., 1997, Anderson et al., 2001; Tamamaki et al., 1997, 2001; Lavdas et al., 1999; Wichterle et al., 1999; Miyata et al., 2001; Noctor et al., 2001; Nery et al., 2002). GABAergic neuron progenitors change from tangential to radial migration after they enter the pallium and move to their final location (Tanaka et al., 2006, 2009; Miyoshi and Fishell, 2011; Bartolini et al., 2013). A majority of cortical GABAergic neuron progenitors in the MGE and POA express Nkx2-1 (Xu et al., 2004; Butt et al., 2005; Wonders and Anderson, 2006; Lim et al., 2018). Fate mapping analyses have shown that the MGE- and POA-derived progenitors are distributed to broad areas of the forebrain (Harwell et al., 2015; García et al., 2016; Mayer et al., 2016; Sultan et al., 2016). Meanwhile, approximately $30 \%$ of all cortical GABAergic neuron progenitors are derived from the CGE, and they are preferentially distributed to the superficial layers (Miyoshi et al., 2010; Miyoshi and Fishell, 2011).

Neural progenitors (also referred to as apical progenitors or radial glia) are located along the ventricles, and apical progenitors of the excitatory neurons produce intermediate progenitors, which divide at the subventricular zone (SVZ) of the dorsal telencephalon to generate two neurons (Noctor et al., 2004; Wu et al., 2005; Pilz et al., 2013). Recently, it was reported that apical progenitors of the inhibitory neurons also produce intermediate progenitors (Wu et al., 2011; Hansen et al., 2013; Petros et al., 2015). Lineage tracing studies have revealed that the Nkx2-1-lineage progenitor cells undergo symmetric and asymmetric division in the SVZ of the MGE (Brown et al., 2011; Ciceri et al., 2013; Sultan et al., 2014). However, the intermediate progenitors of IPGNs have not yet been well characterized. In primates, including humans, many IPGNs are observed at the cortical SVZ as well as the SVZ of the ganglionic eminence at embryonic stages (Letinic et al., 2002; Zecevic et al., 2005, 2011; Petanjek et al., 2009; Jakovcevski et al., 2011; $\mathrm{Yu}$ and Zecevic, 2011), suggesting broad expansion of IPGNs in primates. These IPGNs in the cortical SVZ derive mainly from the outer SVZ of the ganglionic eminence (Hansen et al., 2013; Ma et al., 2013). The CGE, in particular, contributes to a large number of the cortical GABAergic neurons in humans (Hansen et al., 2013).

By contrast, we and others have observed a small number of IPGNs in the cortical-SVZ in embryonic and postnatal mouse brains (Inta et al., 2008; Wu et al., 2011). These IPGNs express neuronal and proliferative markers at the perinatal stage in glutamic acid decarboxylase 67 (GAD67)-GFP knock-in mice, which are widely used to visualize GABAergic neurons and IPGNs. Our previous study revealed that $\sim 1.5 \%$ of the perinatal cortical GAD67-GFP-positive cells self-renew and produce a small number of cortical GABAergic neurons (Wu et al., 2011). Furthermore, a small number of cortical GABAergic neurons are generated in the cortical-SVZ (Inta et al., 2008) and early postnatal dorsal white matter (Riccio et al., 2012). Thus, unlike the intermediate progenitors of the excitatory neurons, IPGNs are widely dispersed from the subpallium to the cerebral cortex. However, the distribution patterns of IPGNs and their fates in adult cerebral cortex are poorly understood.
In this study, we performed systematic analyses of spatiotemporal distribution patterns of IPGNs in mice. Our lineage tracing analyses indicated that the laminar distributions of the embryonic and postnatal IPGNs differ. Late embryonic IPGNs tended to differentiate into reelinpositive $\left(\operatorname{Reln}^{+}\right)$or vasoactive intestinal peptide positive $\left(\mathrm{Vip}^{+}\right)$ GABAergic interneurons but were also able to differentiate into parvalbumin-positive $\left(\mathrm{Pvalb}^{+}\right)$or somatostatin-positive $\left(\mathrm{Sst}^{+}\right)$neurons, suggesting that the IPGNs observed at the late embryonic stage are mainly derived from the CGE, rather than the MGE. Consistently, IPGNs were found primarily in the CGE-SVZ but also the MGE-SVZ and, to lesser extent, in the mantle zone of the ganglionic eminence and cortical-SVZ/intermediate zone (IZ) at embryonic stages. These findings indicate that IPGNs exhibit a unique spatiotemporal distribution and significantly contribute to several subtypes of GABAergic interneurons in adult cerebral cortex.

\section{MATERIALS AND METHODS}

\section{Production of GAD67-CrePR Knock-in Mouse}

Progesterone inducible Cre recombinase (CrePR) was generated to fuse CrePR and progesterone receptor ligand binding motif genes (Kellendonk et al., 1996; Kitayama et al., 2001). To produce GAD67-knock-in CrePR mice, we designed a targeting vector in which a $\mathrm{CrePR}$ recombinase gene was inserted into the translational initiation site of the GAD67 gene in frame (Supplementary Figure 1). A knock-in vector pGAD67CrePRTV contained a $3 \mathrm{~kb}$ fragment at the $5^{\prime}$ side, a CrePR gene placed behind the GAD67 translational start, a $P g k$-neo-p(A) cassette flanked by two Flp recognition target (frt) sites, a $7 \mathrm{~kb}$ fragment at the $3^{\prime}$ side, and an MC1 promoter-driven diphtheria toxin gene. Culture of embryonic stem (ES) cells and generation of chimeric mice were performed as described previously (Kitayama et al., 2001; Higo et al., 2009). Briefly, a linearized pGAD67CrePRTV was introduced into C57BL/6 mouse ES cells (RENKA) and then, G418resistant clones were picked up. Homologous recombined ES clone was identified by Southern blotting. To produce germline chimera, the selected ES cells were microinjected into eight cell-stage embryos of the CD-1 mouse strain. The germline chimeras of GAD67-CrePR mice were crossed with $\mathrm{C} 57 \mathrm{BL} / 6$ mice to generate the GAD67-CrePR mouse line. Because the knock-out of both GAD67 alleles is lethal at birth (Asada et al., 1997), mice heterozygous for the altered GAD67 allele were used for all the observations in this study. Genotypes were identified by Southern blot hybridization or PCR. Tail genomic DNA was digested with Spe-I or Afl-II and hybridized with a $5^{\prime}$ probe or a $3^{\prime}$ probe, respectively. PCR was performed with specific $3^{\prime}$. The sequence of each primer and the approximate length of the amplified DNA fragments are described as follows: Gad1CrePR, g67-2 (5' -TTCCGGAGGTACCACACCTT3'), g67-5 (5' -TAAGTCGACGCTAGCGAGCGCCTCCCCA-3'), 
and CreR1 (5'-TTGCCCCTGTTTCACTATCC-3'); wild type, $1.8 \mathrm{kbp}$; mutant: $1.4 \mathrm{kbp}$.

\section{Animals}

Mice were housed and treated in strict accordance with the rules for animal care and use for research and education of Kumamoto University. They were kept in cages and exposed to a $12 \mathrm{~h}$ light/dark cycle with food and water provided ad libitum. GAD67-CrePR mice express a mifepristone-inducible CrePR under the control of the GAD67 gene. To label $\mathrm{GAD}^{+} 7^{+}$cells, $0.05 \mathrm{mg} / \mathrm{g}$ body weight of mifepristone (SigmaAldrich, St. Louis, MO, United States) was administered to pregnant GAD67-CrePR mice, or $0.05 \mathrm{mg} / \mathrm{g}$ body weight mifepristone was administered to postnatal day 0 pups by intraperitoneal (i.p.) injection. Mifepristone was dissolved in $100 \%$ ethanol to $50 \mathrm{mg} / \mathrm{mL}$ and then diluted in corn oil (Wako, Japan) to $5 \mathrm{mg} / \mathrm{mL}$. To prevent abortions in the pregnant mice, progesterone dissolved in corn oil $(20 \mathrm{mg} / \mathrm{mL}$, stored at $4^{\circ} \mathrm{C}$; Sigma-Aldrich, St. Louis, MO, United States) was administered at $0.15 \mathrm{mg} / \mathrm{g}$ body weight once a day between E12 and E18 (Rose et al., 2009). To detect Cre-mediated recombination in GAD67-CrePR knock-in mice, mice that express TdTomato reporter upon Cre-mediated recombination were used (Jackson stock no. 007909 with an ICR background) (Madisen et al., 2010). GAD67-CrePR knock-in male mice were mated with R26-TdTomato (Ai9) reporter female mice or ICR mice (Oriental Yeast, Japan). As a previous study showed that CreER-mediated reporter recombination is initiated $6 \mathrm{~h}$ after tamoxifen administration, intermediate progenitors of IPGNs and GABAergic neurons are sufficiently labeled with TdTomato $24 \mathrm{~h}$ after the injection (Zervas et al., 2004). RFP antibody were utilized for enhanced visualization of TdTomato expression. Newborn pups were allowed to develop up to 3 weeks. Pregnant mice and juvenile mice (3 weeks) were anesthetized as described below and perfused with a fixative for immunohistochemistry.

\section{Fixation}

The mice were anesthetized with sodium pentobarbital $(50 \mathrm{mg} / \mathrm{kg}$ body weight, i.p.) and perfused transcardially with $10 \mathrm{~mL}$ PBS $[0.9 \%(\mathrm{w} / \mathrm{v})$ saline buffered with $5 \mathrm{mM}$ sodium phosphate, $\mathrm{pH}$ 7.4], followed by $50 \mathrm{~mL}$ PBS containing $4 \%$ (w/v) formaldehyde. The brains were removed, postfixed with the same fixative for $12 \mathrm{~h}$, and then cryoprotected in $25 \%$ sucrose in PBS overnight. The brains were sliced into $50 \mu \mathrm{m}$-thick coronal sections on a cryostat.

\section{Immunohistochemistry}

Immunohistochemistry was performed on the brains of male and/or female mice. Immunohistochemistry was performed as described previously (Tamamaki et al., 2003; Miyamoto and Fukuda, 2015). Briefly, brain sections were rinsed in PBS several times and incubated overnight at room temperature with primary antibody diluted in incubation buffer $(0.3 \%$ Triton $\mathrm{X}-100,5 \%$ donkey serum, and $0.01 \%$ sodium azide in PBS). The primary antibodies used in this experiment are listed in
Table 1. Secondary antibodies were goat anti-rabbit or antirat IgG conjugated with Alexa Fluor 488 and donkey antirabbit IgG conjugated with Alexa Fluor 594 (1:500; Molecular Probes, Eugene, OR, United States), or goat anti-mouse IgM conjugated with Alexa Fluor 594 (1:500; Chemicon, Temecula, CA, United States). The sections were mounted onto MAS-coated glass slides after staining.

\section{EdU Labeling}

To detect proliferative IPGNs, EdU, an analog of the nucleoside thymidine that incorporates into replicating DNA was injected to the GAD67-CrePR;Ai9 mice $24 \mathrm{~h}$ after the mifepristone administration. This inducible genetic fate mapping method enables labeling of postnatally proliferative GAD67 ${ }^{+}$IPGNs and tracing of their lineage in the mature brain. Direct labeling of EdU was performed after incubations with primary antibody. Tissue sections were incubated with $1 \%$ bovine serum albumin in PBS for $1 \mathrm{~h}$ and then with a freshly prepared cocktail containing $50 \mathrm{mM}$ Tris ( $\mathrm{pH} 7.4), 150 \mathrm{mM} \mathrm{NaCl} 2,2 \mathrm{mM}$ $\mathrm{CuSO}_{4}, 10 \mu \mathrm{M}$ Alexa Fluor 647-azide (from $1 \mathrm{mM}$ stock in dimethyl sulfoxide, A10277; Thermo Fisher, Waltham, MA, United States), and $10 \mathrm{mM}$ sodium ascorbate (added last from $0.1 \mathrm{M}$ stock) in the dark for $2 \mathrm{~h}$. Sections were washed in PBS three times (protocol modified from that described by Hua and Kearsey (2011)]. Then, sections were incubated with secondary antibodies.

\section{Quantifications and Statistical Analysis}

EdU-labeled and immunoreactive cells were counted under a confocal microscope (Nikon C2) individually by capturing threedimensional images at a 1-2 $\mu \mathrm{m}$ thickness. Total thickness of three-dimensional images in the depth direction were more than $15 \mu \mathrm{m}$. More than 8 hemisphere of cortical slices and more than 50 cells were analyzed from a brain in each experiment, respectively. Data are presented as means \pm standard errors (SEs). Statistical significance was determined by two-sided unpaired Student's $t$-tests, with a $P$-value of $<0.05$ considered statistically significant.

TABLE 1 | Primary antibodies used in this study.

\begin{tabular}{llll}
\hline Antibody & Dilution & Source or reference & Animal \\
\hline Anti-BrdU & $1: 200$ & Abcam & Rat \\
Anti-GABA & $1: 5,000$ & Sigma & Rabbit \\
Anti-GFP & $1: 500$ & Nacalai Tesque & Rat \\
Anti-Ki67 & $1: 500$ & Epitomics & Rabbit \\
Anti-NeuN & $1: 1,000$ & Chemicon & Mouse \\
Anti-nestin & $1: 200$ & BD Bioscience & Mouse \\
Anti-parvalbumin & $1: 2,000$ & Sigma & Mouse \\
Anti-PCNA & $1: 50$ & Novocastra & Mouse \\
Anti-pHH3 & $1: 200$ & Millipore & Rabbit \\
Anti-somatostatin & $1: 200$ & Millipore & Rabbit \\
Anti-Tuj1 & $1: 500$ & Covance & Mouse \\
Anti-RFP & $1: 500$ & Tomioka et al., 2015 & Rabbit \\
Anti-Vip & $1: 500$ & ImmunoStar & Rabbit
\end{tabular}




\section{RESULTS}

\section{A Small Proportion of GAD67 ${ }^{+}$ Intermediate Progenitors of GABAergic Inhibitory Neurons (IPGNs) Proliferate in Perinatal Cerebral Cortex}

We previously reported the presence of a small number of IPGNs in the SVZ and even in the IZ of the cerebral cortices of GAD67-GFP knock-in mice at the perinatal stage (Wu et al., 2011). However, the number of postnatally generated IPGNs in the cortical-SVZ (or cortical-IZ) in rodents is much lower than that in primates, and it is not known whether IPGNs survive and mature in adult rodent cortex. To assess the distribution of cortical GABAergic neurons generated postnatally from IPGNs, we utilized GAD67-CrePR mice, which expressed CrePR, a mifepristone-inducible Cre, in GABAergic neurons and IPGNs (Figure 1A). To detect the initial population of postnatally proliferating IPGNs in the cortex, we performed immunohistochemistry using GAD67CrePR;Rosa26-floxed-TdTomato (Ai9 line) mice at postnatal day 1 (P1) that had been administered mifepristone at P0 and EdU 30 min before fixation (Figure 1B). GAD67 ${ }^{+}$TdTomato $^{+}$cells were broadly distributed throughout the brain, including the cerebral cortex. Although $\mathrm{EdU}^{+}$cells were mainly located in the neurogenic niches, they could be found throughout the brain, including the superficial layers of the cortex (Figures 1C-E).

A very small number of GAD67 ${ }^{+}$TdTomato $^{+}$cells colabeled with EdU, and these double-positive cells were observed in the cortical SVZ/IZ and cortical marginal zone (Figure 1E). At P1, $0.4 \pm 0.4 \%\left(3 / 615\right.$ cells from three brains) of the GAD67 ${ }^{+}$ TdTomato $^{+}$cells were $\mathrm{EdU}^{+}$, consistent with our previous analyses showing $\mathrm{GAD}^{+} 7^{+}$perinatal IPGNs in the cortex (Wu et al., 2011). Therefore, we defined GAD67 ${ }^{+}$TdTomato $^{+}$ $\mathrm{EdU}^{+}$double-positive cells as IPGNs. Thus, approximately $<0.5 \%$ of labeled cells were postnatally proliferative IPGNs in mouse cortex at P1.

\section{A Small Proportion of GAD67 ${ }^{+}$ Intermediate Progenitors of GABAergic Inhibitory Neurons (IPGNs) Proliferate and Survive in Cerebral Cortex During Perinatal Development}

To compare the fates of proliferative GABAergic neuron progenitors between late embryonic stage and the perinatal stage, we performed a fate mapping analysis. To detect the proliferative GABAergic neuron progenitors during late embryonic stages, EdU was injected into GAD67-CrePR;Ai9 mice from E14.5 to E18.5 (late embryonic stage) or from P1 to P5 (perinatal stage) with mifepristone administered at $\mathrm{P} 0$ to label embryonically or postnatally proliferative IPGNs, respectively (Figure 2A). At 3 weeks after birth, TdTomato $^{+}$cells were broadly distributed throughout the brain, including the cerebral cortex (Figures 2B,C), hippocampus, striatum, thalamus, hypothalamus, midbrain, and cerebellar cortex (Supplementary Figure 2). TdTomato ${ }^{+}$cells were observed in each cortical layer but tended to distribute in the superficial layers (i.e., layers I and II/III) (Figures 2B-D).

To further characterize these $\mathrm{EdU}^{+}$and TdTomato ${ }^{+}$doublepositive GAD67-lineage cells in each layer, we performed immunohistochemical analyses with EdU. When labeled from P1 to $\mathrm{P} 5, \mathrm{EdU}^{+}$cells were broadly distributed throughout the cortex. By contrast, when labeled at the late embryonic stages (from E14.5 to E18.5), EdU ${ }^{+}$cells tended to populate the superficial layers (i.e., layers I and II/III) (Figure 2C). Approximately 51\% [51.2 $\pm 2.8 \%$ (total 336/657 cells from three brains)] of the TdTomato $^{+}$GAD67-lineage cells (labeled at P0) were colabeled with EdU that was injected from E14.5 to E18.5 (Figures 2FH). By contrast, approximately $1.5 \%[1.5 \pm 1.2 \%(13 / 1,046$ cells from six brains)] of the TdTomato ${ }^{+}$GAD67-lineage cells (labeled at P0) were colabeled with EdU that was injected at P1-P5 (Figures 2F-H and Table 2). These results demonstrate that a very small number of postnatal proliferative IPGNs were represented in perinatal cortex by our fate mapping study. Thus, the proliferative rates of the GABAergic neuron progenitors during late embryonic stage were nearly 34-fold higher than that of the postnatally proliferative IPGNs (labeled at P1P5), indicating that significantly more GABAergic neurons are produced during embryonic stages. Moreover, these GAD67 ${ }^{+}$ embryonic proliferating cells (EdU labeled at E14.5-E18.5) were more abundant in the superficial cortical layers (i.e., layers II/III) than in the deep layers (Figures 2F,G and Table 2). These results delineate the fate of later-born embryonic GABAergic neurons and postnatally proliferative IPGNs.

\section{Subtype Specification of Proliferative GAD67-Lineage Cells Derived From Postnatal GAD67+ Intermediate Progenitors of GABAergic Inhibitory Neurons (IPGNs)}

To investigate subtypes of GAD67-lineage cortical GABAergic neurons that were produced during late embryonic stages (EdU administration at E14.5-E18.5) or perinatal stages (EdU administration at P1-P5), we performed immunohistochemistry with subtype markers for $\mathrm{Pvalb}^{+}, \mathrm{Sst}^{+}, \mathrm{Reln}^{+}$, and $\mathrm{Vip}^{+}$ interneurons in conjunction with EdU staining in the GAD67CrePR;Ai9 mice administered mifepristone at P0 to label $\mathrm{GAD}^{+}$cells (Figure 3A). Among the TdTomato ${ }^{+}$GAD67lineage cells, $11.5 \pm 3.8 \%$ were Pvalb $^{+}$(38/329 cells from six brains), $8.9 \pm 4.6 \%$ were $\mathrm{Sst}^{+}$(29/341 cells from six brains), $39.7 \pm 5.6 \%$ were $\operatorname{Reln}^{+}$(126/313 cells from six brains), and $16.6 \pm 7.0 \%$ were $\mathrm{Vip}^{+}$(56/338 cells from six brains) (Figure 3B and Table 3), suggesting that more than $40 \%$ of the GAD67lineage cells labeled at P0 were $\mathrm{Reln}^{+}$or $\mathrm{Vip}^{+}$GABAergic neurons. These results indicate that our fate mapping strategy tends to label later-born CGE-derived GABAergic neurons rather than MGE-derived neurons. Next, we estimated the ratio of the GAD67-lineage cortical GABAergic neurons that were produced during late embryonic stages (EdU labeling at E14.5-E18.5) in each subtype. Among the TdTomato ${ }^{+}$GAD67-lineage cells that were labeled with EdU at E14.5-E18.5, $3.5 \pm 1.7 \%$ (6/172 cells from three brains) were double positive for Pvalb and EdU, 
A

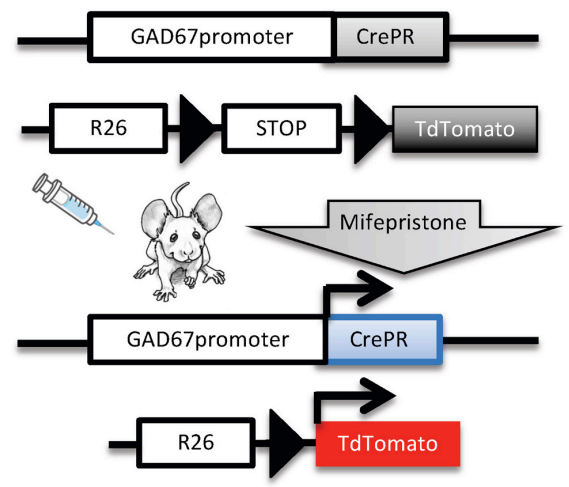

C

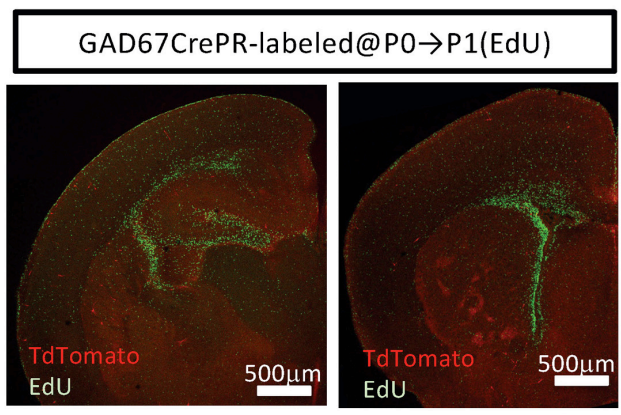

D

\% TdTomato+ (GAD67-lineage) cells labeled@P0 in each cortical region

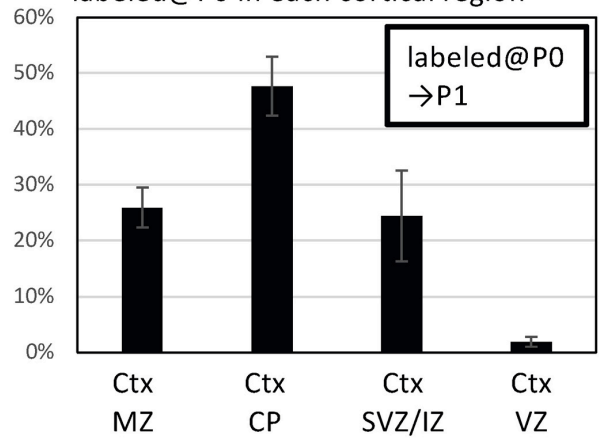

B

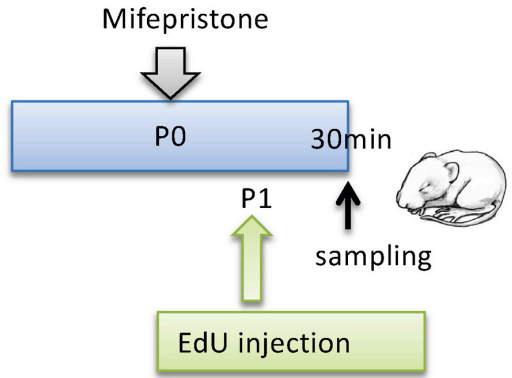

E
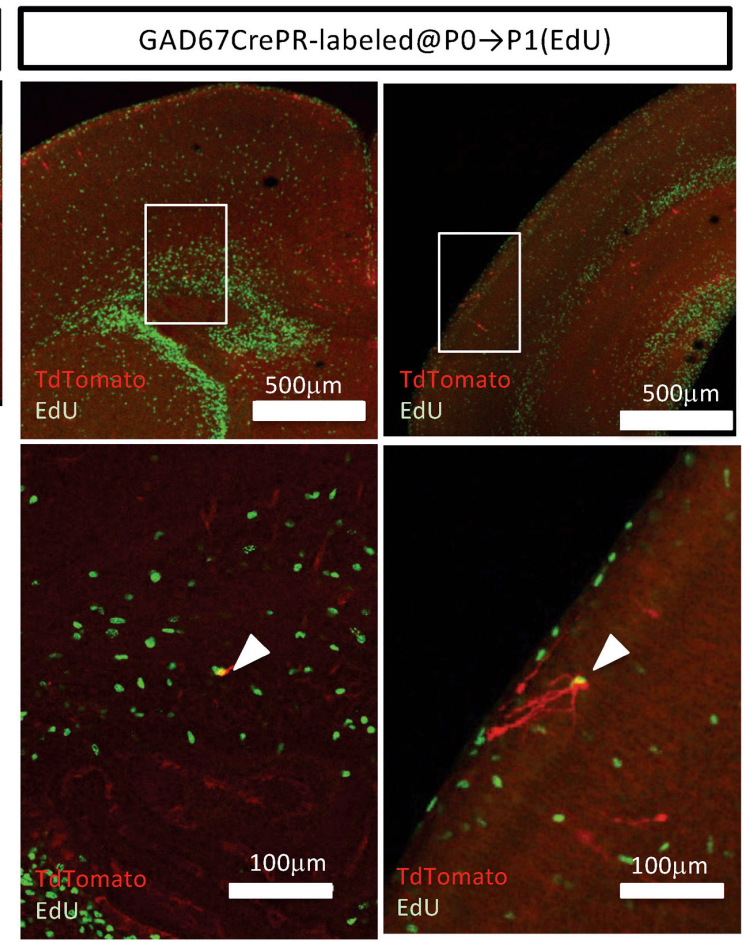

FIGURE 1 | Fate mapping analysis of postnatal GAD67 ${ }^{+}$intermediate progenitors of GABAergic inhibitory neurons (IPGNs) using GAD67-CrePR mouse. (A) Strategy for labeling GAD67-lineage cells by mifepristone-dependent recombination. Cre recombinase is restricted to GAD67-expressing cells after mifepristone treatment. The Rosa26 (R26)-floxed-TdTomato (Ai9) allele expresses TdTomato following Cre-mediated recombination. (B) Experimental design for labeling GAD67-lineage cells at the perinatal stage. Mifepristone was injected i.p. into GAD67-CrePR;Ai9 mice at P0, and EdU was injected at P1. Mifepristone- and EdU-treated mice were analyzed $30 \mathrm{~min}$ after EdU injection. (C) Immunohistochemistry for TdTomato (red) expression and EdU (green) at P1. An anti-RFP antibody was utilized to enhance TdTomato fluorescence. (D) Percentages of TdTomato+ GAD67-lineage cells labeled at P0 in each cortical layer. Ctx, cortex; MZ, marginal zone; CP, cortical plate; SVZ/IZ, subventricular zone/intermediate zone. Error bars represent SDs ( $n=3$ brains). (E) Representative images of TdTomato+ GAD67-lineage cells (red) with EdU (green) in perinatal subventricular zone/intermediate zone (left) and marginal zone (right). Lower images show higher magnification of regions outlined with white boxes. White arrowheads indicate the double-positive cells.

$5.6 \pm 1.6 \%$ ( $9 / 158$ cells from three brains) were double positive for Sst and EdU, $20.6 \pm 2.3 \%$ (32/155 cells from three brains) were double positive for Reln and EdU, and $11.8 \pm 3.0 \%(20 / 172$ cells from three brains) were double positive for Vip and EdU (Figure 3D). These double-positive cells were produced during late embryonic stages and later differentiated into GAD67 ${ }^{+}$
GABAergic neurons at P0. We next investigated the ratio of postnatally proliferative GAD67 ${ }^{+}$IPGNs (EdU labeling at P1P5). However, no double-positive cells were found for Pvalb (total 0/157 cells from three brains), Sst (0/183 cells from three brains), or Reln (0/164 cells from three brains) among the TdTomato $^{+}$GAD67-lineage cells that were labeled with EdU 
A

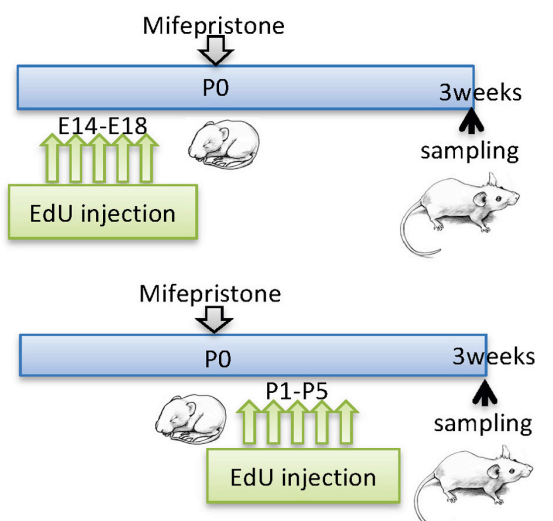

C

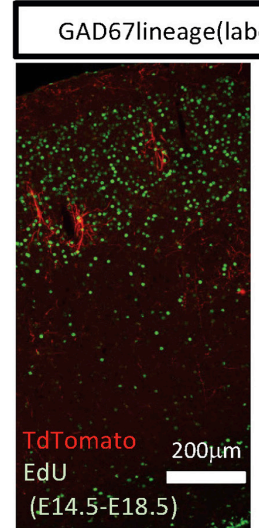

F

\% TdTomato+ cells labeled @P0 (EdU E14-E18) in each layers

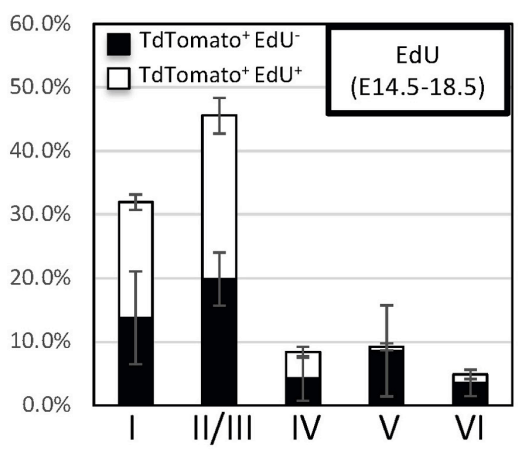

B

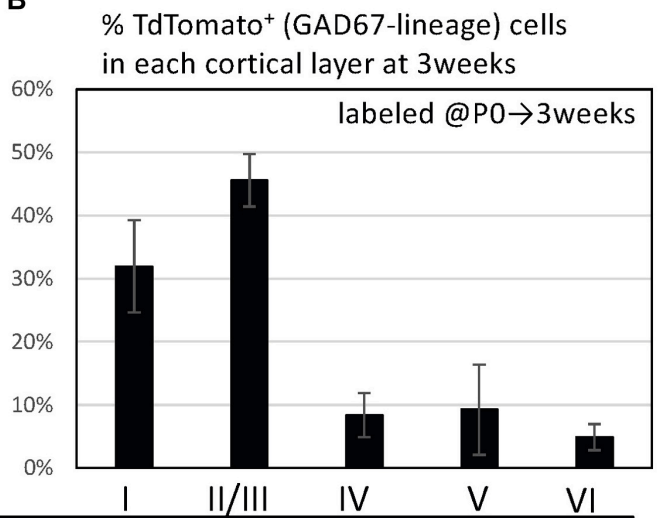

D
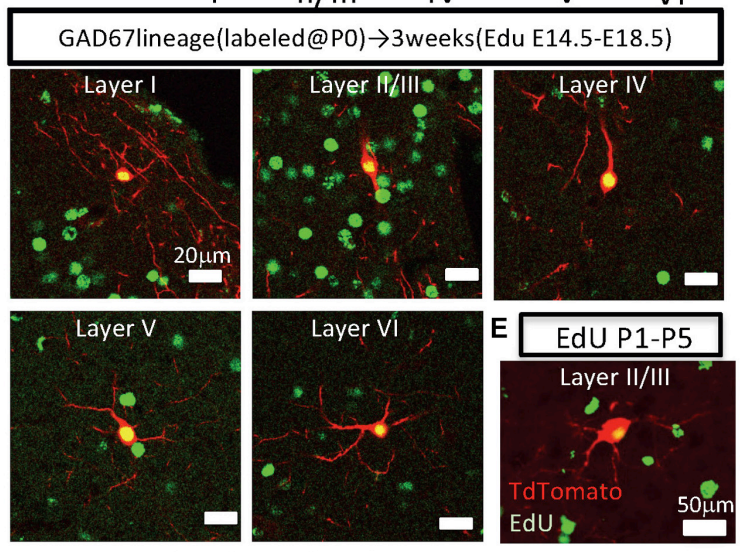

G \% TdTomato ${ }^{+}$cells labeled @P0 (EdU P1-P5) in each layers

\% TdTomato+EdU + (labeled@P0)

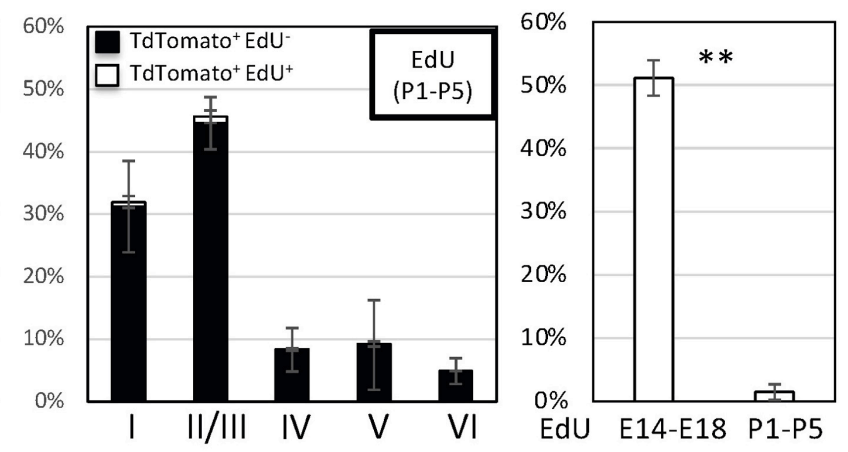

FIGURE 2 | Fate mapping analysis of GAD67+ IPGNs using GAD67-CrePR mouse labeled at P0. (A) Experimental design for labeling IPGNs at the perinatal stage. Mifepristone was administered to pregnant GAD67-CrePR;Ai9 mice at P0, and EdU was injected i.p. from E14.5 to E18.5 or P1 to P5. Mifepristone- and EdU-treated mice were analyzed at 3 weeks of age. IPGNs, intermediate GABAergic neuron progenitors. (B) Percentages of TdTomato+ GAD67-lineage cells in each cortical layer labeled at PO. Error bars represent SDs ( $n=9$ brains). (C) Coronal section of GAD67-CrePR;Ai9 mice brain at 3 weeks with EdU staining (green). EdU was injected E14.5-E18.5 (left) P1-P5 (right) by dual-fluorescence immunohistochemistry. Immunocytochemical analyses were performed with confocal laser microscopy. (D) Representative images of TdTomato+ GAD67-lineage cells labeled at PO (red) with EdU (E14-18) (green) in each cortical layer at 3 weeks of age. (E) Representative image of TdTomato ${ }^{+}$GAD67-lineage cells labeled at PO (red) with EdU (P1-P5) (green) in cortical layer I//II at 3 weeks of age. (F) Percentages of TdTomato $^{+}$cells (GAD67 lineage labeled at P0) labeled with EdU (E14-E18) in each cortical layer. Error bars represent SDs ( $n=3$ brains). (G) Percentages of TdTomato ${ }^{+}$cells (GAD67 lineage labeled at P0) labeled with EdU (P1-P5) in each cortical layer. Error bars represent SDs $(n=6$ brains). (H) Percentages of TdTomato $^{+} \mathrm{EdU}^{+}$GAD67-lineage cells labeled at P0 and EdU injected from E14.5 to E18.5 ( $n=3$ brains) or P1 to P5 $\left(n=6\right.$ brains). ${ }^{* *} P<0.01$. Error bars represent SDs.

injected at the perinatal stage (P1-P5), whereas only $1.6 \pm 1.4 \%$ (2/166 cells from three brains) were double positive for Vip and EdU (Figure 3C). The proportion of cortical GABAergic neurons produced during the late embryonic stage (E14.5E18.5) was significantly higher than that during the perinatal stage $(P<0.01)$ for $\operatorname{Reln}^{+}$and $\mathrm{Vip}^{+}$GABAergic neuron 
TABLE 2 | Localization of GAD67-lineage and EdU+ cells in 3 weeks mice treated with mifepristone at P0 and E13.5.

\begin{tabular}{|c|c|c|c|c|c|c|c|}
\hline \multirow[t]{2}{*}{ Mifepristone } & \multirow[t]{2}{*}{ EdU } & \multicolumn{5}{|c|}{ No. or $\%$ RFP $^{+}$cells in cortical layer } & \multirow[b]{2}{*}{ Total } \\
\hline & & $\mathbf{I}$ & II/III & IV & $\mathbf{v}$ & VI & \\
\hline \multicolumn{8}{|c|}{ No. RFP ${ }^{+}\left(\right.$RFP $^{+}$EdU $\left.^{+}\right)$} \\
\hline E13.5 ( $n=6$ brains) & E14-E18 & $219(86)$ & $291(133)$ & $122(20)$ & 309 (35) & $111(12)$ & 1,052 (286) \\
\hline E13.5 ( $n=3$ brains) & P1-P5 & $115(2)$ & 139 (3) & $75(0)$ & $91(1)$ & $60(0)$ & $480(6)$ \\
\hline PO ( $n=6$ brains $)$ & E14-E18 & $240(118)$ & $283(167)$ & $74(27)$ & $31(4)$ & $22(9)$ & $650(325)$ \\
\hline PO ( $n=3$ brains) & P1-P5 & $294(5)$ & $438(7)$ & $60(1)$ & $80(1)$ & $48(0)$ & $920(14)$ \\
\hline \multicolumn{8}{|l|}{$\%$ RFP $^{+}$} \\
\hline E13.5 ( $n=9$ brains) & & $21.9 \pm 5.4 \%$ & $28.2 \pm 5.0 \%$ & $12.7 \pm 4.9 \%$ & $25.9 \pm 8.5 \%$ & $11.2 \pm 3.3 \%$ & \\
\hline PO ( $n=9$ brains) & & $31.9 \pm 7.3 \%$ & $45.6 \pm 4.2 \%$ & $8.4 \pm 3.5 \%$ & $9.2 \pm 7.2 \%$ & $4.9 \pm 2.1 \%$ & \\
\hline \multicolumn{8}{|l|}{$\% \mathrm{RFP}^{+} \mathrm{EdU}^{+}$} \\
\hline E13.5 ( $n=6$ brains) & E14-E18 & $8.3 \pm 2.4 \%$ & $13.2 \pm 4.9 \%$ & $1.9 \pm 1.1 \%$ & $3.4 \pm 3.5 \%$ & $1.1 \pm 1.1 \%$ & $27.8 \pm 7.5 \%$ \\
\hline E13.5 ( $n=3$ brains) & P1-P5 & $0.4 \pm 0.6 \%$ & $0.6 \pm 0.7 \%$ & $0 \%$ & $0.2 \pm 0.3 \%$ & $0 \%$ & $1.2 \pm 1.1 \%$ \\
\hline PO ( $n=3$ brains) & E14-E18 & $18.2 \pm 1.2 \%$ & $25.7 \pm 2.8 \%$ & $4.1 \pm 0.9 \%$ & $0.6 \pm 0.5 \%$ & $1.4 \pm 0.8 \%$ & $50.0 \pm 2.1 \%$ \\
\hline $\mathrm{PO}(n=6$ brains $)$ & P1-P5 & $0.8 \pm 1.0 \%$ & $1.1 \pm 1.0 \%$ & $0.1 \pm 0.2 \%$ & $0.2 \pm 0.4 \%$ & $0 \%$ & $2.1 \pm 1.8 \%$ \\
\hline
\end{tabular}

subtypes. Interestingly, a small number of GAD67 ${ }^{+}$postnatally proliferative cells colabeled with Vip, a marker for CGE-derived GABAergic neurons (Figure 3D).

\section{Embryonic Proliferation of GAD67 ${ }^{+}$ Intermediate Progenitors of GABAergic Inhibitory Neurons (IPGNs)}

Our data revealed a small number of postnatally proliferative IPGNs (Figures 2, 3). Moreover, the cortical GABAergic neurons that derived from postnatally proliferative IPGNs survived into later postnatal development. On the other hand, significantly more GABAergic neurons that were derived from the GABAergic neuron progenitors were produced during embryonic stages. However, the proportion of the GABAergic neurons derived from the IPGNs in each embryonic stage was unclear. To define the distribution patterns of embryonically proliferating IPGNs, we analyzed E14.5, E16.5, and E18.5 GAD67-CrePR;Ai9 mice that were administered mifepristone at E13.5 to label GAD67 ${ }^{+}$cells, which were sacrificed $30 \mathrm{~min}$ after EdU injection (Figure 4). At E14.5, $3.5 \pm 0.4 \%$ of the TdTomato $^{+}$cells were EdU ${ }^{+}$ (27/793 cells from three brains). At E16.5, $0.6 \pm 0.3 \%$ of the TdTomato $^{+}$cells were $\mathrm{EdU}^{+}$(4/672 cells from three brains). At E18.5, $0.8 \pm 0.2 \%$ of the TdTomato $^{+}$cells were EdU ${ }^{+}$ (5/584 cells from three brains). We found that approximately half of the GAD67-lineage TdTomato $^{+}$cells migrated to the cortex and the other half were in the M/CGE-mantle zone and M/CGE-SVZ at E14.5 (Figures 4A,B). These cells migrated tangentially toward the cerebral cortex via two major migratory streams, a superficial route through the cortical-marginal zone and a deeper route through the cortical-SVZ/IZ, consistent with previous reports (Lavdas et al., 1999; Wichterle et al., 2001; Tanaka et al., 2006, 2009; Miyoshi and Fishell, 2011; Lim et al., 2018). Furthermore, 3.5\% of EdU ${ }^{+}$GAD67-lineage TdTomato $^{+}$double-positive cells were observed in the MGESVZ/IZ or M/CGE-mantle zone at E14.5 (Figures 4A,B). These results suggest that $\sim 3.5 \%$ of the embryonic proliferating IPGNs mainly populated the M/CGE-SVZ/IZ or M/CGE-mantle zone at E14.5. However, no GAD67-lineage TdTomato ${ }^{+}$cells were found in the M/CGE at E18.5. Therefore, migration of the E13.5labeled GAD67-lineage-TdTomato ${ }^{+}$cells from the M/CGE to the cerebral cortex had already finished by E18.5. In addition, a very small proportion $(<1 \%)$ of the GAD67-lineage TdTomato ${ }^{+}$ cells colabeled with EdU in the cortex in the cortical plate and cortical-SVZ/IZ at E16.5 and E18.5 (Figures 4C-F). These results suggest that embryonic brains have multiple regions for embryonic IPGN proliferation. Furthermore, a substantial proportion of the GAD67 ${ }^{+}$IPGNs actively proliferate at around E14.5, and their proliferative activities decline with tangential migration to the cortex.

\section{A Large Proportion of GAD67 ${ }^{+}$ Embryonic IPGNs Proliferate and Survive in Cerebral Cortex During Development}

To determine the ratio of embryonically to postnatally proliferative IPGNs, mifepristone was administered to pregnant GAD67-CrePR;Ai9 mice at E13.5 followed by EdU injections from E14.5 to E18.5 or from P1 to P5 to label proliferative cells (Figure 5A). At 3 weeks after birth, the TdTomato $^{+}$cells labeled by mifepristone administration at E13.5 were broadly distributed throughout the brain, including in the cerebral cortex (Figures 5B-D and Supplementary Figure 3). Interestingly, the distribution pattern of the TdTomato ${ }^{+}$cells labeled at E13.5 was distinct from that observed when mifepristone was injected at P0 (Figures 2B, 5B). The TdTomato ${ }^{+}$cells labeled at E13.5 were observed throughout the cortical layers and tended to accumulate in each cortical layer (Figures 5B-D). To assess the ratio of the $\mathrm{TdTomato}^{+}$GAD67-lineage EdU ${ }^{+}$double-positive cells in each layer, we performed immunohistochemical analyses. The TdTomato ${ }^{+}$GAD67-lineage cells and $\mathrm{EdU}^{+}$cells were detected throughout all cortical layers by immunohistochemistry (Figures 5C,D). The embryonically labeled EdU ${ }^{+}$cells (E14E18) accumulated in the upper layers (i.e., layers II/III), whereas the postnatally labeled EdU ${ }^{+}$cells $(\mathrm{P} 1-\mathrm{P} 5)$ were scattered in each cortical layer. 


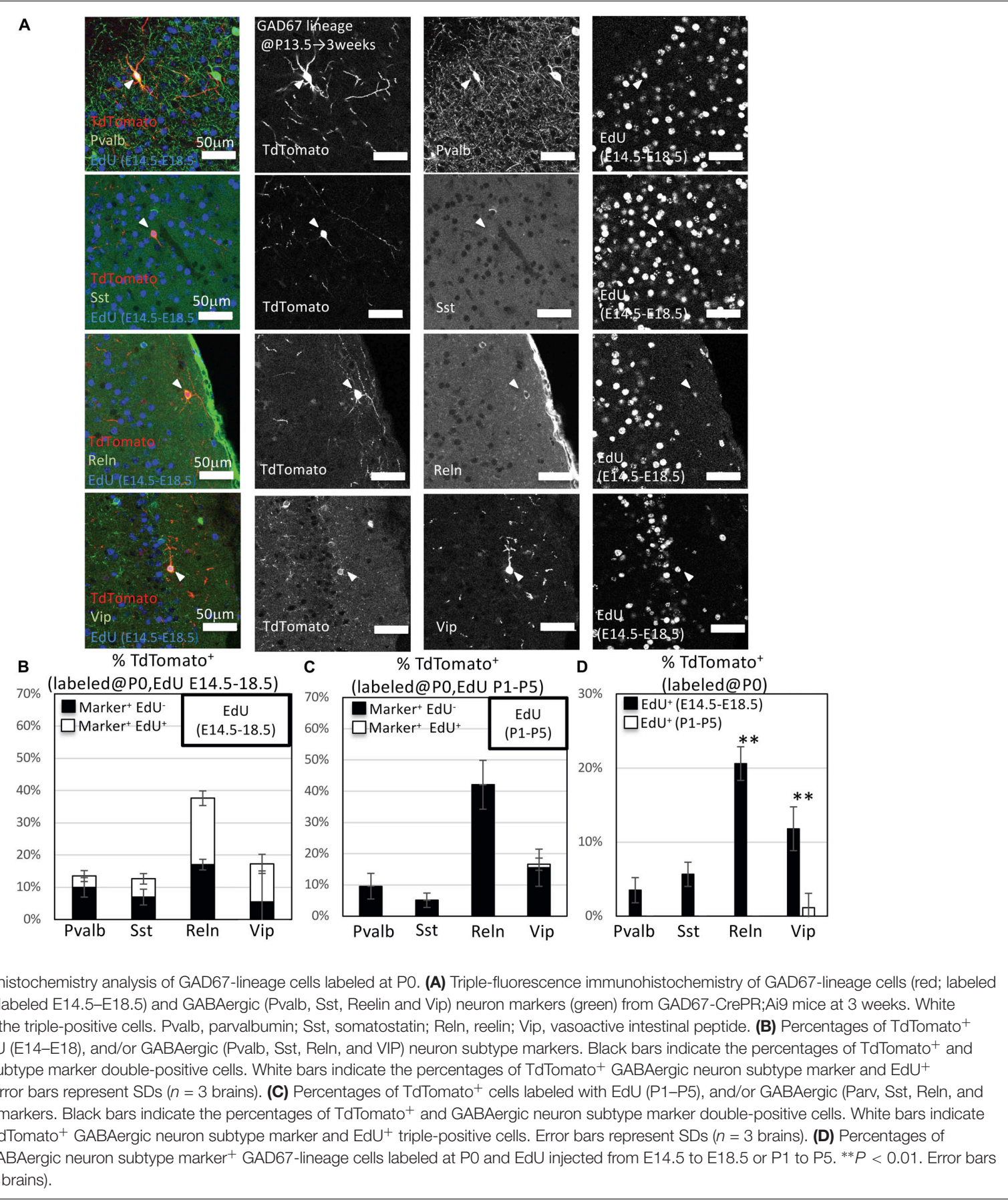

Approximately $26.1 \%[ \pm 6.1 \%(292 / 1,130$ cells from six brains)] (Figures 5F-H) of the TdTomato ${ }^{+}$GAD67-lineage cells (mifepristone administration at E13.5) colabeled with EdU injected from E14.5 to E18.5. By contrast, $1.1 \pm 1.1 \%$ (6/495 cells from three brains) (Figures 5F-H) of the TdTomatopositive GAD67-lineage cells (mifepristone administration at E13.5) colabeled with EdU injected from P1 to P5 (Figure 5G). Thus, the number of embryonically proliferative IPGNs (EdU injection at E14.5-E18.5) was nearly 24-fold higher than that of the postnatally proliferative IPGNs (EdU injection at P1-P5), suggesting that significantly more IPGNs are produced during embryonic stages. These embryonically proliferating cells tended to localize to the superficial layers (i.e., layers II/III) rather than the deep cortical layers at 3 weeks after birth in comparison to the GAD67-lineage cells labeled at P0 (Figures 2F,G, 5F,G and Table 2). By contrast, a very small number of postnatally proliferative IPGNs (mifepristone administration at E13.5 and EdU injection at P1-P5) was observed in the cerebral cortex 3 weeks after birth in our fate mapping study. These results delineate the fate of late embryonic and perinatal proliferative IPGNs. 
TABLE 3 | Percentages of TdTomato+ cells labeled with EdU and/or GABAergic neuron markers.

\begin{tabular}{|c|c|c|c|c|c|}
\hline \multirow[t]{2}{*}{ Mifepristone } & \multicolumn{5}{|c|}{ No. or $\%$ RFP $^{+}$cells in cortex } \\
\hline & EdU & Pvalb & Sst & Reln & Vip \\
\hline \multicolumn{6}{|c|}{ Total no. RFP ${ }^{+}$(Marker $^{+}$RFP ${ }^{+}$) } \\
\hline E13.5 ( $n=6$ brains) & & $312(71)$ & $315(50)$ & $316(135)$ & $321(34)$ \\
\hline PO ( $n=6$ brains $)$ & & $329(38)$ & $341(29)$ & $313(126)$ & $338(56)$ \\
\hline \multicolumn{6}{|l|}{$\%$ Marker $^{+}$RFP $^{+}$} \\
\hline $\mathrm{E} 13.5$ ( $n=6$ brains) & & $22.7 \pm 4.3 \%$ & $15.9 \pm 8.7 \%$ & $42.6 \pm 8.9 \%$ & $10.4 \pm 8.6 \%$ \\
\hline PO ( $n=6$ brains $)$ & & $11.5 \pm 3.8 \%$ & $8.9 \pm 4.6 \%$ & $39.7 \pm 5.6 \%$ & $16.6 \pm 7.0 \%$ \\
\hline \multicolumn{6}{|c|}{ Total No. RFP ${ }^{+}$(Marker $^{+}$RFP $^{+}\left[\right.$Marker $^{+}$RFP $^{+}$EdU $\left.\left.{ }^{+}\right]\right)$} \\
\hline E13.5 ( $n=3$ brains) & E14-E18 & $153(37[5])$ & $153(25[1])$ & $156(57[17])$ & $156(6[4])$ \\
\hline E13.5 ( $n=3$ brains) & P1-P5 & 159 (34 [0]) & $156(25[0])$ & 160 (78 [3]) & $156(28[0])$ \\
\hline PO ( $n=3$ brains) & E14-E18 & $172(23[6])$ & $158(20$ [9]) & $155(58$ [32]) & 172 (29 [20]) \\
\hline PO ( $\mathrm{n}=3$ brains) & P1-P5 & 157 (15 [0]) & 183 (9 [0]) & 158 (68 [0]) & $166(27[2])$ \\
\hline \multicolumn{6}{|c|}{$\%$ Marker $^{+}$RFP $^{+}$EdU $^{+}$} \\
\hline E13.5 ( $n=3$ brains) & E14-E18 & $3.2 \pm 4.0 \%$ & $0.6 \pm 1.1 \%$ & $10.8 \pm 2.6 \%$ & $2.6 \pm 1.2 \%$ \\
\hline E13.5 ( $n=3$ brains) & P1-P5 & $0 \%$ & $0 \%$ & $1.8 \pm 0.4 \%$ & $0 \%$ \\
\hline PO ( $n=3$ brains) & E14-E18 & $3.5 \pm 1.7 \%$ & $5.6 \pm 1.6 \%$ & $20.6 \pm 2.3 \%$ & $11.8 \pm 3.0 \%$ \\
\hline PO ( $n=3$ brains) & P1-P5 & $0 \%$ & $0 \%$ & $0 \%$ & $1.1 \pm 2.0 \%$ \\
\hline
\end{tabular}

\section{Subtype Differentiation (Specification) of Proliferative GAD67-Lineage Cells Derived From Embryonic Intermediate Progenitors of GABAergic Inhibitory Neurons (IPGNs)}

We next performed immunohistochemistry to examine the ratios of $\mathrm{Pvalb}^{+}, \mathrm{Sst}^{+}, \mathrm{Reln}^{+}$, and Vip ${ }^{+}$GABAergic neurons derived from the embryonic IPGNs in GAD67-CrePR;Ai9 mice administered mifepristone at E13.5. At 3 weeks, almost all of the cortical TdTomato $^{+}$cells colabeled with NeuN, a marker for mature neurons, and $>97 \%$ of the cells were positive for gamma-aminobutyric acid (GABA) (Supplementary Figure 4). To investigate subtypes of cortical GABAergic neurons which were produced from embryonic GAD67-lineage IPGNs during late embryonic stages (EdU injection at E14.5-E18.5) or perinatal stages (EdU injection at P1-P5), we performed immunohistochemical analyses of Pvalb, Sst, Reln, and Vip with EdU staining in the GAD67-CrePR;Ai9 mice administered mifepristone at E13.5 to label GAD67 ${ }^{+}$cells (Figure 6A). Among the TdTomato ${ }^{+}$GAD67-lineage cells, $22.7 \pm 4.3 \%$ were Pvalb ${ }^{+}$ (71/312 cells from six brains), $15.9 \pm 8.7 \%$ were Sst $^{+}$(50/315 cells from six brains), $42.6 \pm 8.9 \%$ were Reln ${ }^{+}$(135/316 cells from six brains), and $10.4 \pm 8.6 \%$ were $\mathrm{Vip}^{+}$(34/321 cells from six brains) (Figure 6B and Table 3). These results suggest that GAD67lineage cells that were labeled at E13.5 differentiated into Pvalb ${ }^{+}$, $\mathrm{Sst}^{+}, \mathrm{Reln}^{+}$, and $\mathrm{Vip}^{+}$GABAergic neurons. Interestingly, nearly half of the TdTomato ${ }^{+}$GAD67-lineage cells were Reln $^{+}$. We then estimated the ratio of each GABAergic neuron subtype marker and EdU double-positive cells among the TdTomato ${ }^{+}$ GAD67-lineage cells (EdU injections from E14.5 to E18.5 or from P1 to P5). Among the TdTomato ${ }^{+}$GAD67-lineage cells that were labeled with EdU injected at E14.5-E18.5, $3.2 \pm 4.0 \%$ (5/153 cells from brains) were double positive for Pvalb and EdU, $0.6 \pm 1.1 \%$ ( $1 / 153$ cells from three brains) were double positive for Sst and EdU, $10.8 \pm 2.6 \%$ (17/156 cells from three brains) were double positive for Reln and EdU, and $2.6 \pm 1.2 \%$ (4/156 cells from three brains) were double positive for Vip and EdU (Figure 6D and Table 3). Thus, the GAD67positive IPGNs that proliferated during the late embryonic stages differentiated into $\mathrm{Pvalb}^{+}, \mathrm{Sst}^{+}, \mathrm{Reln}^{+}$, and Vip ${ }^{+}$GABAergic cortical neurons derived from MGE and CGE. In addition, these embryonically proliferative IPGNs preferentially produced $\operatorname{Reln}^{+}$ cortical GABAergic neurons, which may be equivalent to CGEderived GABAergic neurons (Miyoshi and Fishell, 2011). No double-positive cells were found for Pvalb (0/159 cells from three brains), Sst (0/156 cells from three brains), or Vip (0/156 from brains) among the TdTomato ${ }^{+}$GAD67-lineage cells labeled by EdU injection at postnatal stages (P1-P5), whereas only $1.6 \pm 1.4 \%$ (3/160 cells from three brains) were double positive for Reln and EdU (Figures 6C,D). The proportion of the GAD67positive IPGNs that proliferated during the late embryonic stage (E14.5-E18.5) was significantly higher than that during the perinatal stage $(P<0.01)$ for each GABAergic neuron subtype. These results demonstrate that only a very small number of IPGNs postnatally proliferate in the cerebral cortex and that these cells are derived from the CGE.

\section{DISCUSSION}

Cortical GABAergic interneurons comprise approximately $20 \%$ of all neurons (Tamamaki et al., 2003; Sahara et al., 2012) and are generated from apical neural progenitors as well as intermediate progenitors of IPGNs (Wu et al., 2011). The appropriate distribution of GABAergic neurons throughout the neocortex requires not only migratory guidance but also proper regulation of progenitor proliferation. Here, we characterized the spatiotemporal distribution of IPGN-derived cortical GABAergic neurons by using GAD67-CrePR mice, 
A

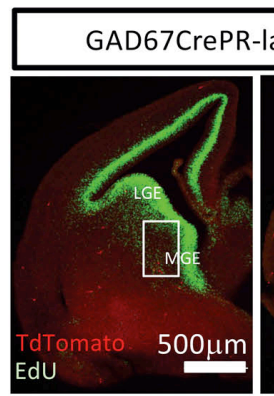

C

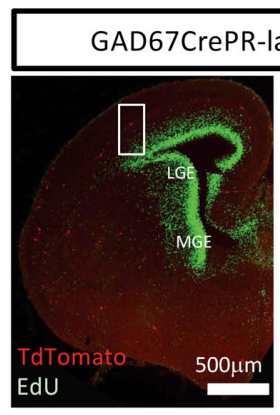

E

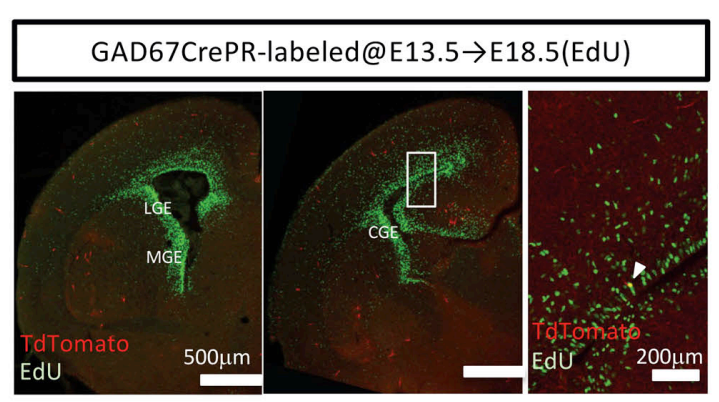

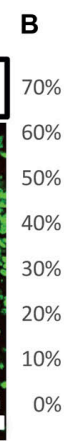

\% TdTomato+ (GAD67-lineage) cells

labeled@E13.5 in each region

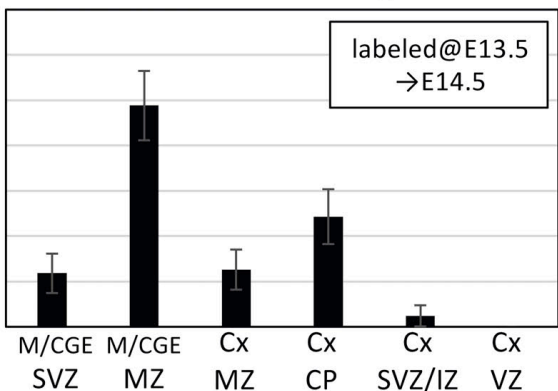

\% TdTomato+ (GAD67-lineage) cells labeled@E13.5 in each region

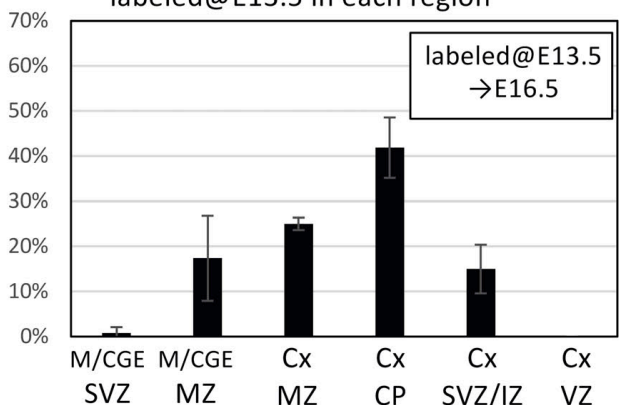

F \% TdTomato ${ }^{+}$(GAD67-lineage) cells labeled@E13.5 in each region

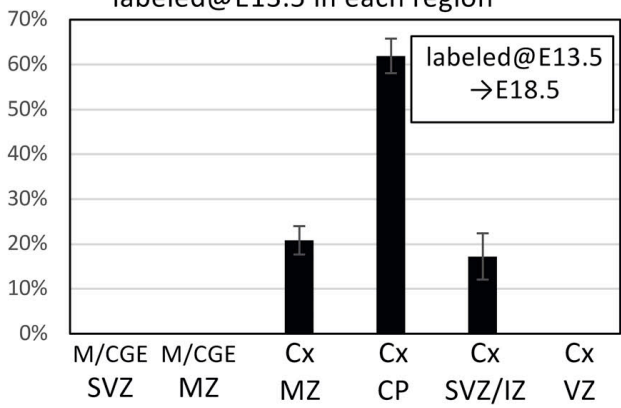

FIGURE 4 | Embryonic proliferation of GAD67-lineage cells. (A) Coronal section of a GAD67-CrePR;Ai9 mouse brain at E14.5 with EdU staining (green). Mifepristone was administered to pregnant GAD67-CrePR;Ai9 mice at E13.5, and EdU was injected i.p. 30 min before fixation. Middle image shows higher magnification of region outlined with white boxes in left column. White arrowhead indicates a double-positive cell in SVZ of MGE. Ctx, cortex; MGE, medial ganglionic eminence; LGE, lateral ganglionic eminence; CGE, caudal ganglionic eminence. (B) Percentages of TdTomato+ cells (GAD67 lineage labeled at E13.5) labeled with EdU (E14.5) in each region. Error bars represent SDs ( $n=3$ brains). Ctx, cortex; M/CGE, medial/caudal ganglionic eminence; MntZ, mantle zone; MrgZ, marginal zone; CP, cortical plate; SVZ/IZ, subventricular zone/intermediate zone; VZ, ventricular zone. (C) Coronal section of a GAD67-CrePR;Ai9 mouse at E16.5 with EdU staining (green). Mifepristone was administered to pregnant GAD67-CrePR;Ai9 mice at E13.5, and EdU was injected i.p. 30 min before fixation. Middle image shows higher magnification of region outlined with white box. White arrowhead indicates a double-positive cell in cortical plate. (D) Percentages of TdTomato+ cells (GAD67 lineage labeled at E13.5) labeled with EdU (E16.5) in each region. Error bars represent SDs ( $n=3$ brains). (E) Coronal section of a GAD67-CrePR;Ai9 mouse brain at E18.5 with EdU staining (green). Mifepristone was administered to pregnant GAD67-CrePR;Ai9 mice at E13.5, and EdU was injected i.p. 30 min before fixation. White arrowhead indicates a double-positive cell in SVZ/IZ. (F) Percentages of TdTomato+ cells (GAD67 lineage labeled at E13.5) labeled with EdU (E18.5) in each region. Error bars represent SDs ( $n=3$ brains).

thereby providing basic and fundamental information toward understanding the mechanisms regulating the development of cortical GABAergic neurons.

Glutamic acid decarboxylase 67 (encoded by Gad1) and GAD65 (encoded by Gad2) are enzymes that catalyze the production of GABA from glutamic acid. While GAD67 and
GAD65 are expressed in mature GABAergic neurons, a small portion of the GAD67/65 cells in the embryonic (M/C) GE and perinatal cortex in rodents and monkeys express proliferative makers (Inta et al., 2008; Wu et al., 2011; Plachez and Puche, 2012; Riccio et al., 2012). These observations indicate that GAD67 and GAD65 are expressed in IPGNs as well as mature 
A

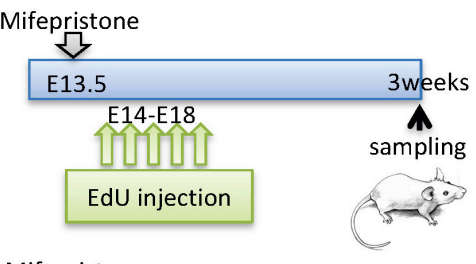

Mifepristone

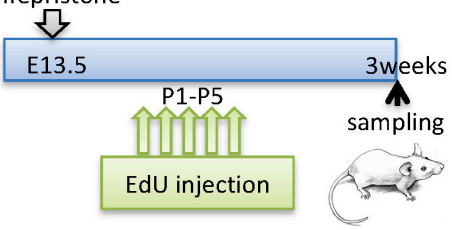

C

GAD67lineage(labeled@E13.5) $\rightarrow$ 3weeks
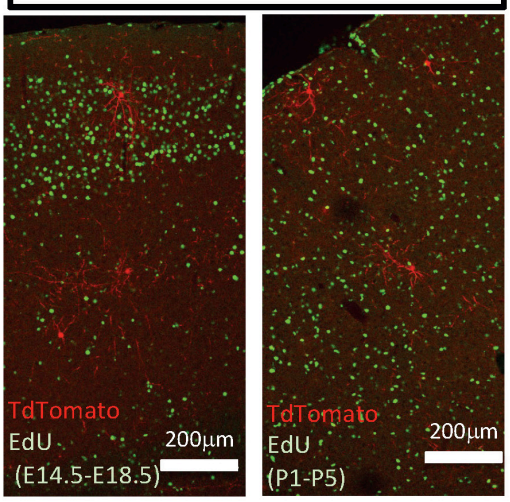

(EdU E14.5-18.5) in each layers

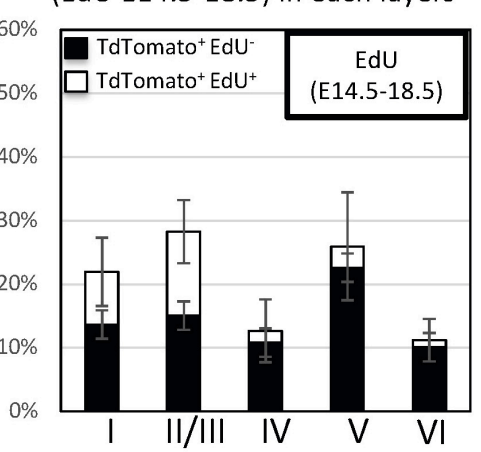

B

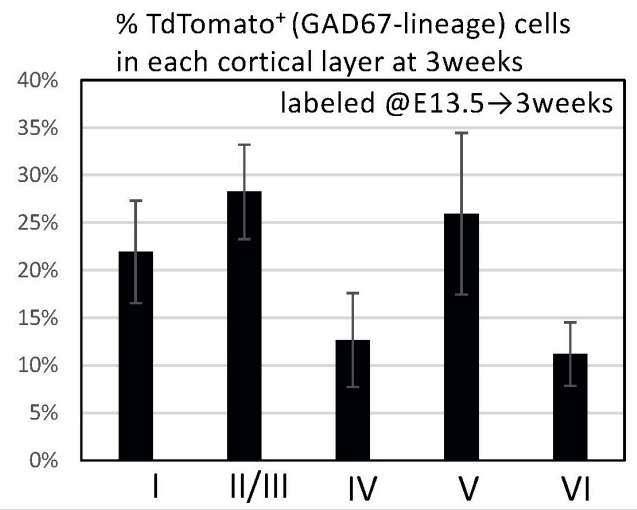

D

GAD67lineage(labeled@E13.5) $\rightarrow$ 3weeks(EdU E14.5-E18.5)
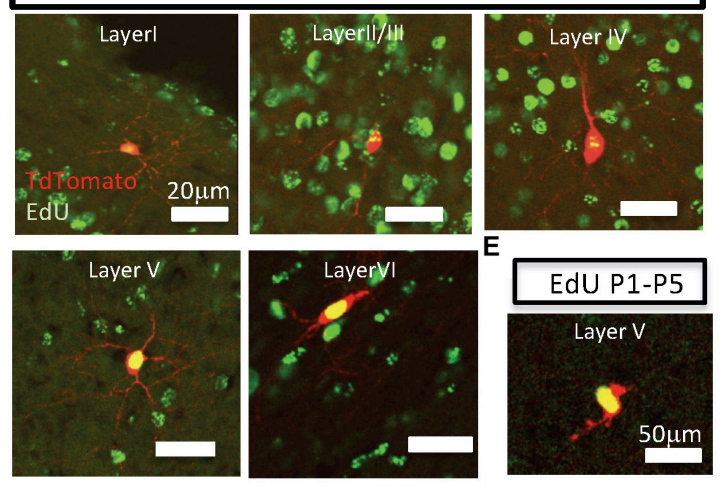

H \% TdTomato EdU $^{+}$

(labeled@E13.5)
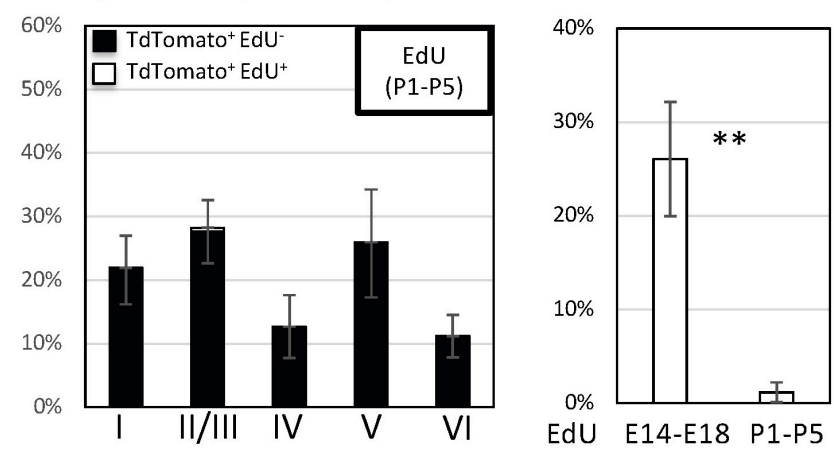

FIGURE 5 | Fate mapping analysis of GAD67+ IPGNs in GAD67-CrePR mice labeled at E13.5. (A) Experimental design for labeling IPGNs at the embryonic stage. Mifepristone was administered to pregnant GAD67-CrePR;Ai9 mice at E13.5, and EdU was injected i.p. from E14.5 to E18.5 or P1 to P5. Mifepristone- and EdU-treated mice were analyzed at 3 weeks. (B) Percentages of TdTomato+ GAD67-lineage cells labeled at E13.5 in each cortical layer. Error bars represent SDs ( $n=9$ brains). (C) Coronal section of GAD67-CrePR;Ai9 mouse labeled at E13.5 at 3 weeks with EdU staining (green). EdU was injected E14.5-E18.5 (left) P1-P5 (right) by dual-fluorescence immunohistochemistry. (D) Representative images of TdTomato ${ }^{+}$GAD67-lineage cells labeled at E13.5 with EdU (E14-18) (green) in each cortical layer at 3 weeks. (E) Representative image of TdTomato ${ }^{+}$GAD67-lineage cells with EdU (P1-P5) (green) in cortical layer $V$ at 3 weeks. (F) Percentages of TdTomato ${ }^{+}$cells labeled at E13.5 with EdU (E14-E18) in each cortical layer. Error bars represent SDs ( $n=6$ brains). (G) Percentages of TdTomato ${ }^{+}$cells labeled at E13.5 with EdU (P1-P5) in each cortical layer. Error bars represent SDs ( $n=6$ brains). (H) Percentages of TdTomato+ EdU ${ }^{+}$GAD67-lineage cells labeled at P0 and EdU injected from E14.5 to E18.5 ( $n=6$ brains) or P1 to P5 ( $n=3$ brains). ${ }^{*} P<0.01$. Error bars represent SDs.

GABAergic neurons in rodents and monkeys. Consistent with this, Dlx2, a transcription factor expressed by IPGNs (Hansen et al., 2013; Petros et al., 2015), binds to the promoter regions of Gad1 and Gad2 (Le et al., 2017). Furthermore, gene expression profiling, single-cell RNA-sequencing, and microarray analyses using cortical GABAergic neurons and/or their progenitors have revealed that $\mathrm{GAD}^{+} 7^{+}$cells can transition from apical progenitors to differentiated mature cortical GABAergic neurons (Esumi et al., 2008; Mayer et al., 2018; Mi et al., 2018). Thus, proliferative GAD67 ${ }^{+}$cells should be 
A
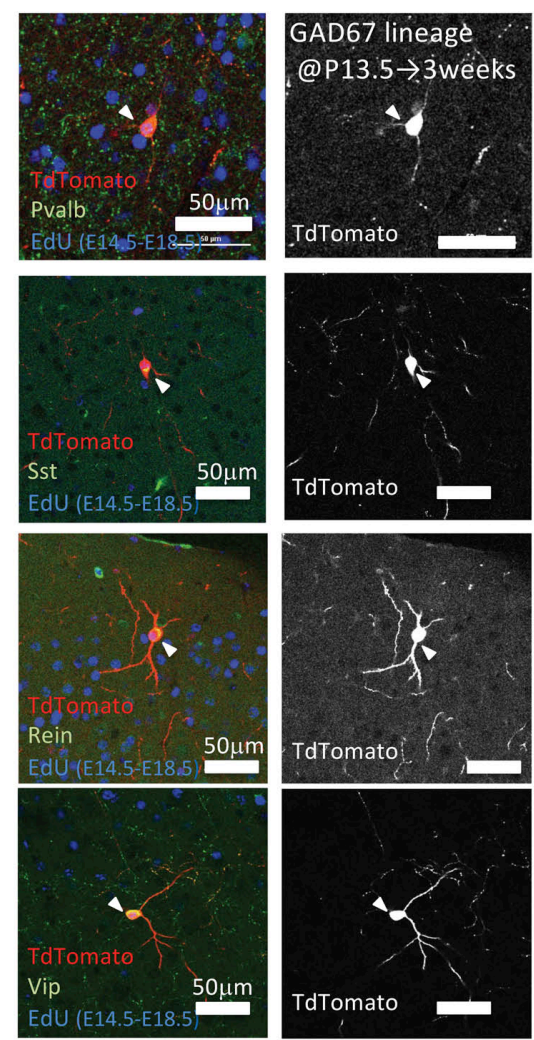

$\%$ TdTomato $^{+}$
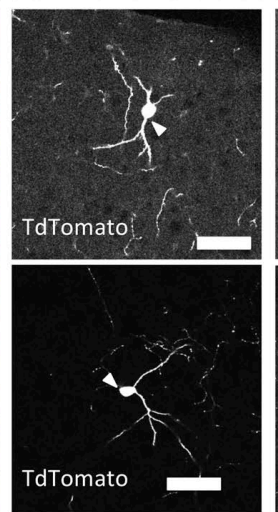

Reln
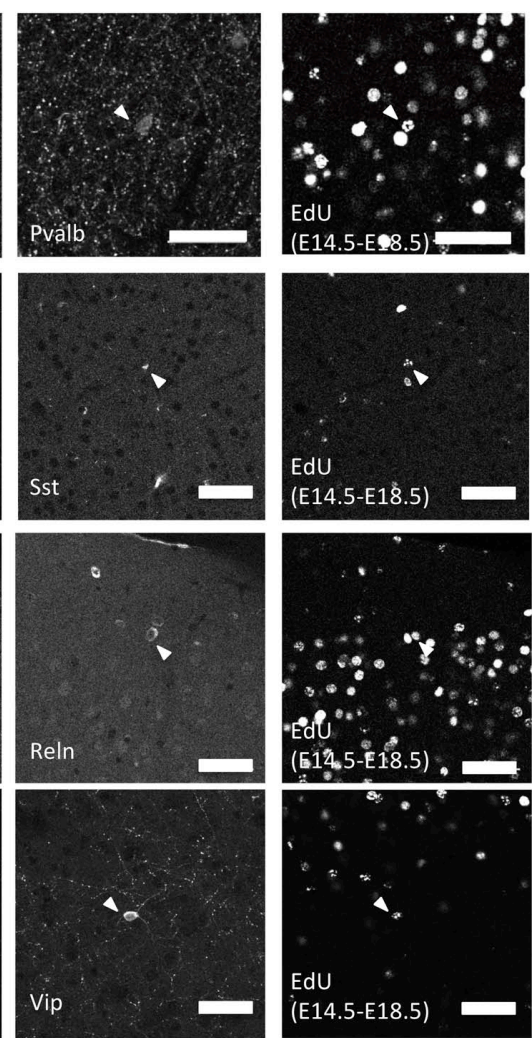

$\%$ TdTomato ${ }^{+}$
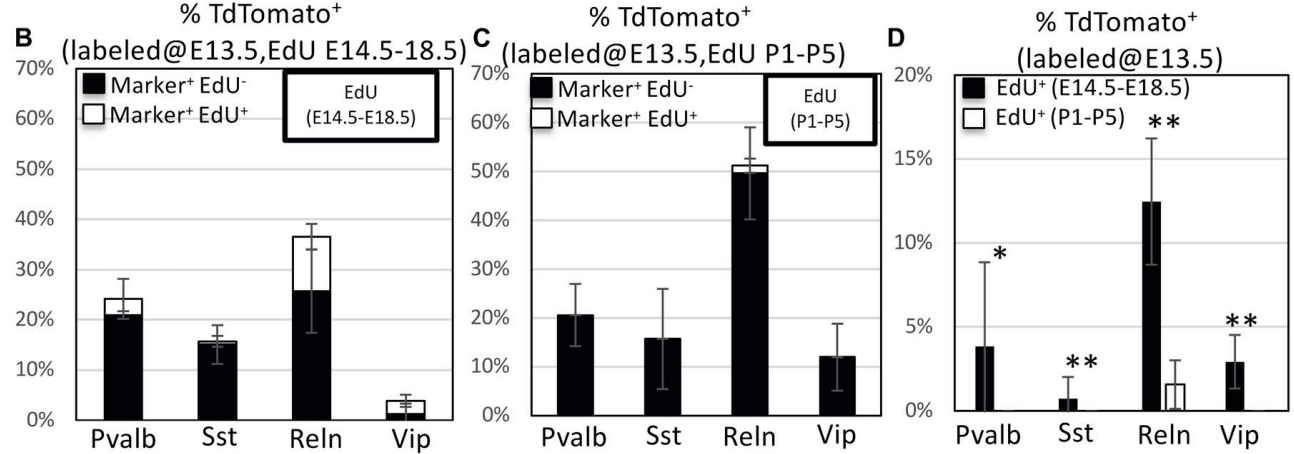

FIGURE 6 | Immunohistochemistry analysis of GAD67-lineage cells labeled at E13.5. (A) Triple-fluorescence immunohistochemistry of GAD67-lineage cells (red; labeled at E13.5) for EdU (green; labeled E14.5-E18.5) and GABAergic (Pvalb, Sst, Reln, and Vip) neuron markers (green) from GAD67-CrePR;Ai9 mice at 3 weeks of age. White arrowheads indicate the triple-positive cells. Pvalb, parvalbumin; Sst, somatostatin; Reln, reelin; Vip, vasoactive intestinal peptide. (B) Percentage of TdTomato ${ }^{+}$cells labeled with EdU (E14-E18), and/or GABAergic (Pvalb, Sst, Reln, and Vip) neuron subtype markers. Black bars indicate the percentages of

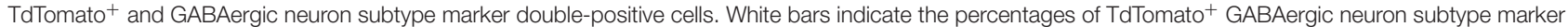
and EdU+ triple-positive cells. (C) Percentage of TdTomato ${ }^{+}$cells labeled with EdU (P1-P5) and/or GABAergic (Pvalb, Sst, Reln, and Vip) neuron subtype markers. Black bars indicate the percentages of TdTomato ${ }^{+}$and GABAergic neuron subtype marker double-positive cells. White bar indicates the percentage of TdTomato ${ }^{+}$ GABAergic neuron subtype marker and EdU + triple-positive cells. (D) Percentages of TdTomato ${ }^{+}$EdU $^{+}$GABAergic neuron subtype marker ${ }^{+}$GAD67-lineage cells labeled at E13.5 and EdU injected from E14.5 to E18.5 or P1 to P5. ${ }^{*} P<0.05 ;{ }^{* *} P<0.01$.

considered late-state intermediate/basal progenitors of cortical GABAergic neurons.

Our fate mapping analyses revealed that the GAD67-lineage cells labeled at P0 tended to distribute to the superficial cortical layers compared to those labeled at E13.5 (Figure 2B). Approximately $70 \%$ of cortical GABAergic neurons reportedly derive from the MGE, whereas $\sim 30 \%$ derive from the CGE, and early-born GABAergic neurons tend to locate to the deeper layers of rodent cortex, whereas late-born GABAergic neurons are found in superficial layers (Miller, 1985; Fairén et al., 1986; Rymar and Sadikot, 2007; Bartolini et al., 2013).

Cortical GABAergic neurons can be classified as $\mathrm{Pvalb}^{+}$, $\mathrm{Sst}^{+}, \mathrm{Reln}^{+}$, and Vip ${ }^{+}$(Miyoshi et al., 2010; Brandão and Romcy-Pereira, 2015). Whereas $\mathrm{Sst}^{+}$neurons are produced during the early stage of neurogenesis, a large proportion of the $\mathrm{Pvalb}^{+}$neurons are generated in the MGE during the late 
stages (Rymar and Sadikot, 2007). On the other hand, the CGEderived GABAergic neurons are produced in late embryonic stages and mainly express either Reln or Vip (Nery et al., 2002; Miyoshi et al., 2010; Rudy et al., 2011; Vitalis and Rossier, 2011). Reln ${ }^{+}$neurons are derived from both the CGE and MGE, as subpopulations of the MGE-derived $\mathrm{Sst}^{+}$neurons coexpress Reln (Miyoshi et al., 2010; Miyoshi and Fishell, 2011). Recently, a number of the CGE-derived GABAergic neurons, which express Reln and Vip, were found to populate superficial cortical layers independently of their birthdate (Miyoshi et al., 2010), and their peak production was during late embryonic stages (Miyoshi and Fishell, 2011). In primates, the IPGNs (basal/non-epithelial progenitors) derived from CGE contribute to a large number of cortical GABAergic neurons (Hansen et al., 2013). These previous reports indicate that the specification of the GABAergic neuron subtypes depends on their birthdates and localization.

Our data indicated that embryonically proliferative GAD67lineage cells (labeled at E13.5) tend to populate the superficial cortical layers. From the view of proliferative IPGNs (labeled at E13.5), approximately $63 \%$ expressed Reln, and $\sim 15 \%$ of those were Vip, but only $<1 \%$ were labeled Sst (Figure 6F). Our results indicate that more than half of cortical IPGNs during late embryonic stage are derived from the CGE. This tendency may correspond to primate cortical GABAergic neuron development.

Several lines of evidence indicate that GABAergic neuron progenitors in the MGE produce both $\mathrm{Pvalb}^{+}$and $\mathrm{Sst}^{+}$cortical GABAergic neurons (Brown et al., 2011; Ciceri et al., 2013; Harwell et al., 2015; Mayer et al., 2015). Sst ${ }^{+}$GABAergic neurons are principally produced from early-stage GABAergic neuron progenitors $\left(\sim\right.$ E12.5), whereas Pvalb $^{+}$GABAergic neurons are continuously produced throughout development (Miyoshi et al., 2007; Wonders et al., 2008; Inan et al., 2012). However, these reports did not distinguish apical (epithelial) progenitors that divide in the ventricular zone (VZ) from IPGNs (basal/nonepithelial progenitors) that divide in the SVZ (Pilz et al., 2013). Basal/non-epithelial progenitors in the SVZ of the MGE mainly produce $\mathrm{Pvalb}^{+}$rather than $\mathrm{Sst}^{+}$cortical GABAergic neurons (Petros et al., 2015). Accordingly, our immunohistochemical analyses showed that GAD67 ${ }^{+}$IPGNs that derive from MGE $\left(\mathrm{Pvalb}^{+}\right.$or $\mathrm{Sst}^{+}$) and travel along the migratory stream to the cortex in late embryonic stages (E14-E18) tend to become Pvalb ${ }^{+}$ neurons, although a small proportion become $\mathrm{Sst}^{+}$neurons. Thus, GAD67 ${ }^{+}$IPGNs in embryonic stages derived from the MGE are most likely to have and retain characteristics of latestage basal progenitors.

Cortical GABAergic neurons are produced from cortical layer 1 GABAergic neuron progenitor cells (L1-INP cells) (Ohira et al., 2010), and the generation of L1-INP cells increases under ischemic conditions and after treatment with fluoxetine, a selective serotonin reuptake inhibitor (Ohira et al., 2013). We observed that a small number of postnatal proliferative GAD67 ${ }^{+}$IPGNs that were labeled after mifepristone administration at E13.5 and P0 survived throughout postnatal development and tended to populate the superficial cortical layers (Figures 2G, 5G). These results suggest that GAD67 ${ }^{+}$
IPGNs are maintained in the cortex and may act as L1-INP cells in adult brain.

In conclusion, the present study demonstrates that GAD67 ${ }^{+}$ IPGNs, which are considered late-stage basal progenitors of cortical GABAergic neurons, substantially contribute to the GABAergic neuron population in adult cerebral cortex. In addition, the proliferative rates of IPGNs and the laminar distributions of their progenies change during cortical development. Thus, the characteristic features of IPGNs are spatiotemporally regulated during brain development.

\section{DATA AVAILABILITY STATEMENT}

The original contributions presented in the study are included in the article/Supplementary Material, further inquiries can be directed to the corresponding author/s.

\section{ETHICS STATEMENT}

The animal study was reviewed and approved by the rules for animal care and use for research and education of Kumamoto University.

\section{AUTHOR CONTRIBUTIONS}

All authors listed have made a substantial, direct and intellectual contribution to the work, and approved it for publication. SE, NT, and TF coordinated the project. KS generated GAD67-CrePR mice. TS and YY provided transgenic mice. SE conceived and designed the study and performed experiments, collected data, and co-wrote the manuscript with TK, MN, KoM, KeM, TS and YY helped to perform the experiments. All authors have seen and agreed with the content of the manuscript.

\section{FUNDING}

This work was supported by the Grant-in-Aid for Scientific Research KAKENHI (20700291, 24700327, 16K08470, and 20K07227), Takeda Science Foundation, and the Japan Epilepsy Research Foundation (JERF TENKAN 18002).

\section{ACKNOWLEDGMENTS}

We gratefully acknowledge Kenji Shimamura for good discussion and technical advice. We would like to thank Ryohei Tomioka for providing antibodies. We would also like to thank Yuri Itoh for technical support. We would like to thank to Dr. Katie Sokolowski for drawing mouse pictures.

\section{SUPPLEMENTARY MATERIAL}

The Supplementary Material for this article can be found online at: https://www.frontiersin.org/articles/10.3389/fnins.2021. 607908/full\#supplementary-material 


\section{REFERENCES}

Anderson, S. A., Eisenstat, D. D., Shi, L., and Rubenstein, J. L. (1997). Interneuron migration from basal forebrain to neocortex: dependence on Dlx genes. Science 278, 474-476. doi: 10.1126/science.278.5337.474 doi: 10.1126/science.278.5337. 474

Anderson, S. A., Marín, O., Horn, C., Jennings, K., and Rubenstein, J. L. (2001). Distinct cortical migrations from the medial and lateral ganglionic eminences. Development 128, 353-363. doi: 10.1093/cercor/bhx185

Asada, H., Kawamura, Y., Maruyama, K., Kume, H., Ding, R. G., Kanbara, N., et al. (1997). Cleft palate and decreased brain gamma-aminobutyric acid in mice lacking the $67-\mathrm{kDa}$ isoform of glutamic acid decarboxylase. Proc. Natl. Acad. Sci. U.S.A. 94, 6496-6499. doi: 10.1073/pnas.94.12.6496

Bartolini, G., Ciceri, G., and Marín, O. (2013). Integration of GABAergic interneurons into cortical cell assemblies: lessons from embryos and adults. Neuron 79, 849-864. doi: 10.1016/j.neuron.2013.08.014

Bozzi, Y., Casarosa, S., and Caleo, M. (2012). Epilepsy as a neurodevelopmental disorder. Front. Psychiatry 3:19. doi: 10.3389/fpsyt.2012.00019

Brandão, J. A., and Romcy-Pereira, R. N. (2015). Interplay of environmental signals and progenitor diversity on fate specification of cortical GABAergic neurons. Front. Cell Neurosci. 9:149. doi: 10.3389/fncel.2015.00149

Brown, K. N., Chen, S., Han, Z., Lu, C. H., Tan, X., Zhang, X. J., et al. (2011). Clonal production and organization of inhibitory interneurons in the neocortex. Science 334, 480-486. doi: 10.1126/science.1208884

Butt, S. J., Fuccillo, M., Nery, S., Noctor, S., Kriegstein, A., Corbin, J. G., et al. (2005). The temporal and spatial origins of cortical interneurons predict their physiological subtype. Neuron 48, 591-604. doi: 10.1016/j.neuron.2005.09.034

Ciceri, G., Dehorter, N., Sols, I., Huang, Z. J., Maravall, M., and Marín, O. (2013). Lineage-specific laminar organization of cortical GABAergic interneurons. Nat. Neurosci. 16, 1199-1210. doi: 10.1038/nn.3485

Esumi, S., Wu, S. X., Yanagawa, Y., Obata, K., Sugimoto, Y., and Tamamaki, N. (2008). Method for single-cell microarray analysis and application to geneexpression profiling of GABAergic neuron progenitors. Neurosci. Res. 60, 439-451. doi: 10.1016/j.neures.2007.12.011

Fairén, A., Cobas, A., and Fonseca, M. (1986). Times of generation of glutamic acid decarboxylase immunoreactive neurons in mouse somatosensory cortex. J. Comp. Neurol. 251, 67-83. doi: 10.1002/cne.902510105

García, T. M., Mazzola, E., and Harwell, C. C. (2016). Lineage relationships do not drive MGE/PoA-derived interneuron clustering in the brain. Neuron 92, 52-58. doi: 10.1016/j.neuron.2016.09.034

Hansen, D. V., Lui, J. H., Flandin, P., Yoshikawa, K., Rubenstein, J. L., AlvarezBuylla, A., et al. (2013). Non-epithelial stem cells and cortical interneuron production in the human ganglionic eminences. Nat. Neurosci. 16, 1576-1587. doi: $10.1038 / \mathrm{nn} .3541$

Harwell, C. C., Fuentealba, L. C., Gonzalez-Cerrillo, A., Parker, P. R., Gertz, C. C., Mazzola, E., et al. (2015). Wide dispersion and diversity of clonally related inhibitory interneurons. Neuron 87, 999-1007. doi: 10.1016/j.neuron.2015.07. 030

Higo, S., Akashi, K., Sakimura, K., and Tamamaki, N. (2009). Subtypes of GABAergic neurons project axons in the neocortex. Front. Neuroanat. 3:25. doi: 10.3389/neuro.05.025.2009 doi: 10.3389/neuro.05.025.2009

Hua, H., and Kearsey, S. E. (2011). Monitoring DNA replication in fission yeast by incorporation of 5-ethynyl-2' -deoxyuridine. Nucleic Acids Res. 39:e60. doi: 10.1093/nar/gkr063

Inan, M., Welagen, J., and Anderson, S. A. (2012). Spatial and temporal bias in the mitotic origins of somatostatin- and parvalbumin-expressing interneuron subgroups and the chandelier subtype in the medial ganglionic eminence. Cereb. Cortex 22, 820-827. doi: 10.1093/cercor/bhr148

Inta, D., Alfonso, J., von Engelhardt, J., Kreuzberg, M. M., Meyer, A. H., van Hooft, J. A., et al. (2008). Neurogenesis and widespread forebrain migration of distinct GABAergic neurons from the postnatal subventricular zone. Proc. Natl. Acad. Sci. U S A. 105, 20994-20999. doi: 10.1073/pnas.0807059105

Jakovcevski, I., Mayer, N., and Zecevic, N. (2011). Multiple origins of human neocortical interneurons are supported by distinct expression of transcription factors. Cereb. Cortex 21, 1771-1782. doi: 10.1093/cercor/bhq245

Kellendonk, C., Tronche, F., Monaghan, A. P., Angrand, P. O., Stewart, F., and Schütz, G. (1996). Regulation of Cre recombinase activity by the synthetic steroid RU 486. Nucleic Acids Res. 24, 1404-1411. doi: 10.1093/nar/24.8.1404
Kitayama, K., Abe, M., Kakizaki, T., Honma, D., Natsume, R., Fukaya, M., et al. (2001). Purkinje cell-specific and inducible gene recombination system generated from C57BL/6 mouse ES cells. Biochem. Biophys. Res. Commun. 281, 1134-1140. doi: 10.1006/bbrc.2001.4492

Lavdas, A. A., Grigoriou, M., Pachnis, V., and Parnavelas, J. G. (1999). The medial ganglionic eminence gives rise to a population of early neurons in the developing cerebral cortex. J. Neurosci. 19, 7881-7888. doi: 10.1523/ JNEUROSCI.19-18-07881.1999

Le, T. N., Zhou, Q. P., Cobos, I., Zhang, S., Zagozewski, J., Japoni, S., et al. (2017). GABAergic interneuron differentiation in the basal forebrain is mediated through direct regulation of glutamic acid Decarboxylase Isoforms by Dlx homeobox transcription factors. J. Neurosci. 37, 8816-8829. doi: 10.1523/ JNEUROSCI.2125-16.2017

Letinic, K., Zoncu, R., and Rakic, P. (2002). Origin of GABAergic neurons in the human neocortex. Nature 417, 645-649. doi: 10.1038/nature00779

Lim, L., Mi, D., Llorca, A., and Marín, O. (2018). Development and functional diversification of cortical interneurons. Neuron 100, 294-313. doi: 10.1016/j. neuron.2018.10.009

Ma, T., Wang, C., Wang, L., Zhou, X., Tian, M., Zhang, Q., et al. (2013). Subcortical origins of human and monkey neocortical interneurons. Nat. Neurosci. 16, 1588-1597. doi: 10.1038/nn.3536

Madisen, L., Zwingman, T. A., Sunkin, S. M., Oh, S. W., Zariwala, H. A., Gu, H., et al. (2010). A robust and high-throughput Cre reporting and characterization system for the whole mouse brain. Nat. Neurosci. 13, 133-140. doi: 10.1038/nn. 2467

Marín, O. (2012). Interneuron dysfunction in psychiatric disorders. Nat. Rev. Neurosci. 13, 107-120. doi: 10.1038/nrn3155

Mayer, C., Bandler, R. C., and Fishell, G. (2016). Lineage is a poor predictor of interneuron positioning within the forebrain. Neuron 92, 45-51. doi: 10.1016/j. neuron.2016.09.035

Mayer, C., Hafemeister, C., Bandler, R. C., Machold, R., Batista Brito, R., Jaglin, X., et al. (2018). Developmental diversification of cortical inhibitory interneurons. Nature 555, 457-462.

Mayer, C., Jaglin, X. H., Cobbs, L. V., Bandler, R. C., Streicher, C., Cepko, C. L., et al. (2015). Clonally related forebrain interneurons disperse broadly across both functional areas and structural boundaries. Neuron 87, 989-998. doi: 10. 1016/j.neuron.2015.07.011

Mi, D., Li, Z., Lim, L., Li, M., Moissidis, M., Yang, Y., et al. (2018). Early emergence of cortical interneuron diversity in the mouse embryo. Science 360, 81-88. doi: $10.1038 /$ nature25999

Miller, M. W. (1985). Cogeneration of retrogradely labeled corticocortical projection and GABA-immunoreactive local circuit neurons in cerebral cortex. Brain Res. 355, 187-192. doi: 10.1016/0165-3806(85)90040-9

Miyamoto, Y., and Fukuda, T. (2015). Immunohistochemical study on the neuronal diversity and three-dimensional organization of the mouse entopeduncular nucleus. Neurosci. Res. 94, 37-49. doi: 10.1016/j.neures. 2015.02.006

Miyata, T., Kawaguchi, A., Okano, H., and Ogawa, M. (2001). Asymmetric inheritance of radial glial fibers by cortical neurons. Neuron 31, 727-741. doi: 10.1016/s0896-6273(01)00420-2

Miyoshi, G., Butt, S. J., Takebayashi, H., and Fishell, G. (2007). Physiologically distinct temporal cohorts of cortical interneurons arise from telencephalic Olig2-expressing precursors. J. Neurosci. 27, 7786-7798. doi: 10.1523/ JNEUROSCI.1807-07.2007

Miyoshi, G., and Fishell, G. (2011). GABAergic interneuron lineages selectively sort into specific cortical layers during early postnatal development. Cereb. Cortex 21, 845-852. doi: 10.1093/cercor/bhq155

Miyoshi, G., Hjerling-Leffler, J., Karayannis, T., Sousa, V. H., Butt, S. J., Battiste, J., et al. (2010). Genetic fate mapping reveals that the caudal ganglionic eminence produces a large and diverse population of superficial cortical interneurons. J. Neurosci. 30, 1582-1594. doi: 10.1523/JNEUROSCI.451509.2010

Nery, S., Fishell, G., and Corbin, J. G. (2002). The caudal ganglionic eminence is a source of distinct cortical and subcortical cell populations. Nat. Neurosci. 5, 1279-1287. doi: 10.1038/nn971

Noctor, S. C., Flint, A. C., Weissman, T. A., Dammerman, R. S., and Kriegstein, A. R. (2001). Neurons derived from radial glial cells establish radial units in neocortex. Nature 409, 714-720. doi: 10.1038/35055553 
Noctor, S. C., Martínez-Cerdeño, V., Ivic, L., and Kriegstein, A. R. (2004). Cortical neurons arise in symmetric and asymmetric division zones and migrate through specific phases. Nat. Neurosci. 7, 136-144. doi: 10.1038/nn1172

Ohira, K., Furuta, T., Hioki, H., Nakamura, K. C., Kuramoto, E., Tanaka, Y., et al. (2010). Ischemia-induced neurogenesis of neocortical layer 1 progenitor cells. Nat. Neurosci. 13, 173-179. doi: 10.1038/nn.2473

Ohira, K., Takeuchi, R., Iwanaga, T., and Miyakawa, T. (2013). Chronic fluoxetine treatment reduces parvalbumin expression and perineuronal nets in gammaaminobutyric acidergic interneurons of the frontal cortex in adult mice. Mol. Brain. 6:43. doi: 10.1186/1756-6606-6-43

Petanjek, Z., Berger, B., and Esclapez, M. (2009). Origins of cortical GABAergic neurons in the cynomolgus monkey. Cereb. Cortex. 19, 249-262. doi: 10.1093/ cercor/bhn078

Petros, T. J., Bultje, R. S., Ross, M. E., Fishell, G., and Anderson, S. A. (2015). Apical versus basal neurogenesis directs cortical interneuron subclass fate. Cell Rep. 13, 1090-1095. doi: 10.1016/j.celrep.2015.09.079

Pilz, G. A., Shitamukai, A., Reillo, I., Pacary, E., Schwausch, J., Stahl, R., et al. (2013). Amplification of progenitors in the mammalian telencephalon includes a new radial glial cell type. Nat. Commun. 4:2125. doi: 10.1038/ncomms3125

Plachez, C., and Puche, A. C. (2012). Early specification of GAD67 subventricular derived olfactory interneurons. J. Mol. Histol. 43, 215-221. doi: 10.1007/s10735012-9394-2

Riccio, O., Murthy, S., Szabo, G., Vutskits, L., Kiss, J. Z., Vitalis, T., et al. (2012). New pool of cortical interneuron precursors in the early postnatal dorsal white matter. Cereb. Cortex 22, 86-98. doi: 10.1093/cercor/bhr086

Rose, M. F., Ahmad, K. A., Thaller, C., and Zoghbi, H. Y. (2009). Excitatory neurons of the proprioceptive, interoceptive, and arousal hindbrain networks share a developmental requirement for Math1. Proc. Natl. Acad. Sci. U S A. 106, 22462-22467. doi: 10.1073/pnas.0911579106

Rudy, B., Fishell, G., Lee, S., and Hjerling-Leffler, J. (2011). Three groups of interneurons account for nearly $100 \%$ of neocortical GABAergic neurons. Dev. Neurobiol. 71, 45-61. doi: 10.1002/dneu.20853

Rymar, V. V., and Sadikot, A. F. (2007). Laminar fate of cortical GABAergic interneurons is dependent on both birthdate and phenotype. J. Comp. Neurol. 501, 369-380. doi: 10.1002/cne.21250

Sahara, S., Yanagawa, Y., O'Leary, D. D., and Stevens, C. F. (2012). The fraction of cortical GABAergic neurons is constant from near the start of cortical neurogenesis to adulthood. J. Neurosci. 32, 4755-4761. doi: 10.1523/ JNEUROSCI.6412-11.2012

Sohal, V. S., and Rubenstein, J. L. (2019). Excitation-inhibition balance as a framework for investigating mechanisms in neuropsychiatric disorders. Mol. Psychiatry. 24, 1248-1257. doi: 10.1038/s41380-019-0426-0

Sultan, K. T., Han, Z., Zhang, X. J., Xianyu, A., Li, Z., Huang, K., et al. (2016). Clonally related GABAergic interneurons do not randomly disperse but frequently form local clusters in the forebrain. Neuron 92, 31-44. doi: 10.1016/j.neuron.2016.09.033

Sultan, K. T., Shi, W., and Shi, S. H. (2014). Clonal origins of neocortical interneurons. Curr. Opin. Neurobiol. 26, 125-131. doi: 10.1016/j.conb.2014.01. 010

Tamamaki, N., Fujimori, K. E., and Takauji, R. (1997). Origin and route of tangentially migrating neurons in the developing neocortical intermediate zone. J. Neurosci. 17, 8313-8323. doi: 10.1523/JNEUROSCI.17-21-08313.1997

Tamamaki, N., Nakamura, K., Okamoto, K., and Kaneko, T. (2001). Radial glia is a progenitor of neocortical neurons in the developing cerebral cortex. Neurosci. Res. 41, 51-60. doi: 10.1016/s0168-0102(01)00259-0

Tamamaki, N., Yanagawa, Y., Tomioka, R., Miyazaki, J. I, Obata, K., and Kaneko, T. (2003). Greenfluorescent protein expression and colocalization with calretinin, parvalbumin and somatostatin in the GAD67-GFP knock-in mouse. J. Comp.Neurol. 467, 60-79. doi: 10.1002/cne.10905

Tanaka, D. H., Maekawa, K., Yanagawa, Y., Obata, K., and Murakami, F. (2006). Multidirectional and multizonal tangential migration of GABAergic interneurons in the developing cerebral cortex. Development 133, 2167-2176. doi: 10.1242/dev.02382

Tanaka, D. H., Yanagida, M., Zhu, Y., Mikami, S., Nagasawa, T., Miyazaki, J., et al. (2009). Random walk behavior of migrating cortical interneurons in the marginal zone: time-lapse analysis in flat-mount cortex. J. Neurosci. 29, 1300-1311. doi: 10.1523/JNEUROSCI.5446-08.2009

Tomioka, R., Sakimura, K., and Yanagawa, Y. (2015). Corticofugal GABAergic projection neurons in the mouse frontal cortex. Front. Neuroanat. 9:133. doi: 10.3389/fnana.2015.00133 doi: 10.3389/fnana.2015.00133

Vitalis, T., and Rossier, J. (2011). New insights into cortical interneurons development and classification: contribution of developmental studies. Dev. Neurobiol. 71, 34-44. doi: 10.1002/dneu.20810

Wichterle, H., Garcia-Verdugo, J. M., Herrera, D. G., and Alvarez-Buylla, A. (1999). Young neurons from medial ganglionic eminence disperse in adult and embryonic brain. Nat. Neurosci. 2, 461-466. doi: 10.1038/8131 doi: 10.1038/ 8131

Wichterle, H., Turnbull, D. H., Nery, S., Fishell, G., and Alvarez-Buylla, A. (2001). In utero fate mapping reveals distinct migratory pathways and fates of neurons born in the mammalian basal forebrain. Development 128, 37593771.

Wonders, C. P., and Anderson, S. A. (2006). The origin and specification of cortical interneurons. Nat. Rev. Neurosci. 7, 687-696. doi: 10.1038/nrn 1954

Wonders, C. P., Taylor, L., Welagen, J., Mbata, I. C., Xiang, J. Z., and Anderson, S. A. (2008). A spatial bias for the origins of interneuron subgroups within the medial ganglionic eminence. Dev. Biol. 314, 127-136. doi: 10.1016/j.ydbio.2007. 11.018

Wu, S. X., Esumi, S., Watanabe, K., Chen, J., Nakamura, K. C., Nakamura, K., et al. (2011). Tangential migration and proliferation of intermediate progenitors of GABAergic neuronsi in the mouse telencephalon. Development 138, 2499-2509. doi: 10.1242/dev.063032

Wu, S. X., Goebbels, S., Nakamura, K., Nakamura, K., Kometani, K., Minato, N., et al. (2005). Pyramidal neurons of upper cortical layers generated by NEXpositive progenitor cells in the subventricular zone. Proc. Natl. Acad. Sci. U S A. 102, 17172-17177. doi: 10.1073/pnas.0508560102

Xu, Q., Cobos, I., De La Cruz, E., Rubenstein, J. L., and Anderson, S. A. (2004). Origins of cortical interneuron subtypes. J. Neurosci. 24, 2612-2622. doi: 10. 1523/JNEUROSCI.5667-03.2004

Yu, X., and Zecevic, N. (2011). Dorsal radial glial cells have the potential to generate cortical interneurons in human but not in mouse brain. J. Neurosci. 31, 2413-2420. doi: 10.1523/JNEUROSCI.5249-10.2011

Zecevic, N., Chen, Y., and Filipovic, R. (2005). Contributions of cortical subventricular zone to the development of the human cerebral cortex. J. Comp. Neurol. 491, 109-122. doi: 10.1002/cne.20714

Zecevic, N., Hu, F., and Jakovcevski, I. (2011). Interneurons in the developing human neocortex. Dev. Neurobiol. 71, 18-33. doi: 10.1002/dneu.20812

Zervas, M., Millet, S., Ahn, S., and Joyner, A. L. (2004). Cell behaviors and genetic lineages of the mesencephalon and rhombomere 1. Neuron 43, 345-357. doi: 10.1016/j.neuron.2004.07.010

Conflict of Interest: The authors declare that the research was conducted in the absence of any commercial or financial relationships that could be construed as a potential conflict of interest.

Copyright (c) 2021 Esumi, Nasu, Kawauchi, Miike, Morooka, Yanagawa, Seki, Sakimura, Fukuda and Tamamaki. This is an open-access article distributed under the terms of the Creative Commons Attribution License (CC BY). The use, distribution or reproduction in other forums is permitted, provided the original author(s) and the copyright owner(s) are credited and that the original publication in this journal is cited, in accordance with accepted academic practice. No use, distribution or reproduction is permitted which does not comply with these terms. 